\title{
Glycolytic inhibitor 2-deoxyglucose prevents cortical hyperexcitability after traumatic brain injury
}

\author{
Jenny B. Koenig, ${ }^{1,2}$ David Cantu, ${ }^{1}$ Cho Low, ${ }^{1,3}$ Mary Sommer, ${ }^{1}$ Farzad Noubary, ${ }^{4}$ Danielle Croker, ${ }^{1}$ \\ Michael Whalen, ${ }^{5,6}$ Dong Kong, ${ }^{1}$ and Chris G. Dulla ${ }^{1}$ \\ 'Department of Neuroscience, Tufts University School of Medicine, Boston, Massachusetts, USA. \\ ${ }^{2}$ Neuroscience Program, Tufts University Sackler School of Graduate Biomedical Sciences, Boston, Massachusetts, USA. \\ ${ }^{3}$ Cellular, Molecular, and Developmental Biology Program, Tufts University Sackler School of Graduate Biomedical \\ Sciences, Boston, Massachusetts, USA. ${ }^{4}$ Department of Health Sciences, Bouvé College of Health Sciences, Northeastern \\ University, Boston, Massachusetts, USA. ${ }^{5}$ Neuroscience Center, Harvard Medical School, Massachusetts Ceneral Hospital, \\ Charlestown, Massachusetts, USA. ${ }^{6}$ Department of Pediatrics, Harvard Medical School, Massachusetts General Hospital, \\ Boston, Massachusetts, USA.
}

Traumatic brain injury (TBI) causes cortical dysfunction and can lead to posttraumatic epilepsy. Multiple studies demonstrate that GABAergic inhibitory network function is compromised following TBI, which may contribute to hyperexcitability and motor, behavioral, and cognitive deficits. Preserving the function of GABAergic interneurons, therefore, is a rational therapeutic strategy to preserve cortical function after TBI and prevent long-term clinical complications. Here, we explored an approach based on the ketogenic diet, a neuroprotective and anticonvulsant dietary therapy that results in reduced glycolysis and increased ketosis. Utilizing a pharmacologic inhibitor of glycolysis (2-deoxyglucose, or 2-DC), we found that acute in vitro application of 2-DC decreased the excitability of excitatory neurons, but not inhibitory interneurons, in cortical slices from naive mice. Employing the controlled cortical impact (CCI) model of TBI in mice, we found that in vitro 2-DC treatment rapidly attenuated epileptiform activity seen in acute cortical slices 3-5 weeks after TBI. One week of in vivo 2-DG treatment immediately after TBI prevented the development of epileptiform activity, restored excitatory and inhibitory synaptic activity, and attenuated the loss of parvalbumin-expressing inhibitory interneurons. In summary, 2-DG may have therapeutic potential to restore network function following TBI.

Conflict of interest: The authors have declared that no conflict of interest exists.

Copyright: @ 2019 American Society for Clinical Investigation

Submitted: November 29, 2018

Accepted: April 23, 2019

Published: June 6, 2019.

Reference information: /CI Insight. 2019;4(11):e126506. https://doi. org/10.1172/ji.insight.126506

\section{Introduction}

Traumatic brain injury (TBI) is a leading cause of death and disability worldwide and can lead to motor, behavioral, and cognitive losses (1) as well as posttraumatic epilepsy (PTE) (2-4). Patients who develop PTE show higher mortality than TBI patients without epilepsy, and many PTE patients are refractory to the currently available anticonvulsive therapies $(2,4)$. Multiple molecular and cellular changes that occur after TBI may play an important role in the pathophysiology of network dysfunction, PTE, and other TBI-induced pathologies. Aberrant electrical activity $(5,6)$, indiscriminate release of neurotransmitters (7), and altered cerebral glucose utilization $(8,9)$ all occur after TBI. Additionally, compromised cerebral blood flow, disruption of the blood-brain barrier $(10,11)$, increased oxidative stress, and mitochondrial dysfunction (12) create a complex set of neurometabolic disruptions after injury $(1,2,13)$. Together, these changes can disturb synaptic function, cause hard-wired remodeling of neuronal connectivity, and lead to circuit dysfunction and PTE.

Many studies suggest that a key step in developing network dysfunction after injury is the loss of inhibitory GABAergic interneurons, a diverse group of neurons that powerfully constrain cortical excitation. Disruption of inhibitory network activity can lead to motor dysfunction, cognitive losses, and epilepsy (14-18). The most abundant subtype of cortical interneurons express the calcium-binding protein parvalbumin (PV), fire action potentials at a high frequency (fast-spiking), and provide perisomatic inhibition of excitatory cortical pyramidal neurons (19). $\mathrm{PV}^{+}$interneuron loss, atrophy, and dysfunction have been shown in the controlled cortical impact (CCI) (20), lateral fluid percussion (14), partially isolated 
neocortex ("undercut") $(21,22)$, and weight drop (23) models of TBI. Importantly, human postmortem studies also show that PV-expressing interneurons are specifically lost following TBI (24). The mechanisms by which TBI leads to interneuron loss are largely unknown. Therefore, it has been difficult to develop therapeutic interventions to preserve inhibitory network function following TBI.

We believe that the aberrant neuronal activity that occurs acutely after TBI is a key contributor to longterm cortical network dysfunction. In support of this idea, early seizure activity and other EEG abnormalities are associated with poor clinical outcomes and a higher risk of developing posttraumatic epilepsy $(5,6$, 25). Preclinical data from animal models of epilepsy and brain injury demonstrate that early uncontrolled excitation contributes to later loss of GABAergic interneurons and development of inhibitory circuit dysfunction (26-29). Following TBI in both humans and animal models, this aberrant electrical activity occurs during periods of abnormally elevated glycolytic activity (30-35). We hypothesize that reducing hyperglycolysis will attenuate injury-induced increases in excitatory synaptic activity, ameliorate losses of $\mathrm{PV}^{+}$ interneurons, and ultimately prevent inhibitory network dysfunction.

2-Deoxyglucose (2-DG) is a glucose analog that competitively inhibits hexokinase (the rate-limiting enzyme in glycolysis) (36-38). Multiple studies demonstrate that 2-DG can be anticonvulsive in both in vitro and in vivo models of epilepsy $(39,40)$. 2-DG pretreatment also prevents kainic acid-induced hippocampal cell loss in rats (41) and can reduce seizure severity $(42,43)$. While 2 -DG has been previously explored as an anticonvulsive therapy, it has not yet been utilized as a disease-modifying agent to prevent epileptogenesis after TBI.

In this study, we tested whether 2-DG reduces TBI-induced hyperexcitation and attenuates the development of inhibitory network dysfunction after TBI. We utilized the CCI model of focal brain contusion, which results in significant cell loss, network hyperexcitability, and spontaneous behavioral and electrographic seizures (44). Our study shows that acute 2-DG treatment of cortical brain sections attenuated hyperexcitability in the injured brain. In addition, 2-DG treatment reduced the excitability of excitatory neurons, but not inhibitory interneurons. Finally, in vivo treatment with 2-DG for 1 week following CCI prevented the development of epileptiform activity and reduced the loss of PV-expressing interneurons. Together, these studies support the potential application of glycolytic inhibitors to reduce cortical hyperexcitability after TBI.

\section{Results}

In vitro 2-DG treatment decreases the excitability of excitatory neurons, but not inhibitory interneurons. 2-DG has been previously shown to attenuate epileptiform activity in multiple in vitro and animal models of epilepsy $(39,40,45)$. Pharmacologic inhibition of glycolysis can directly suppress synaptic transmission, reduce membrane resistance (46), and broaden the action potential (AP) waveform (47). It is unknown, however, whether glycolytic inhibition has differential effects on the excitability of glutamatergic excitatory neurons and GABAergic inhibitory interneurons. Therefore, we assessed the effects of 2-DG on both cell types in naive (uninjured) cortex. Whole-cell recordings were established in acute cortical brain slices from adult (>P28) animals of both sexes. Recordings were performed on layer V pyramidal neurons or fast-spiking $\mathrm{PV}^{+}$GABAergic interneurons. $\mathrm{PV}^{+}$interneurons were identified using the $G 42$ mouse line, a BAC transgenic line in which greater than $70 \%$ of genetically GFP-labeled interneurons are $\mathrm{PV}^{+}$on immunohistochemistry (Supplemental Figure 1 and ref. 48; supplemental material available online with this article; https:// doi.org/10.1172/jci.insight.126506DS1). In current-clamp mode, hyperpolarizing and depolarizing steps were injected in the presence of synaptic receptor blockers $(10 \mu \mathrm{m}$ CPP, $20 \mu \mathrm{m}$ DNQX, and $10 \mu \mathrm{m}$ gabazine) and the number of evoked APs was quantified. The results were compared between baseline artificial cerebrospinal fluid (aCSF, containing $10 \mathrm{mM}$ glucose) and following 10 minutes of slice perfusion with 2-DG-aCSF (8 mM 2-DG, $2 \mathrm{mM}$ glucose). 2-DG-aCSF maintained the same solution osmolarity as baseline aCSF (10 mM total saccharide), while limiting glucose availability to concentrations similar to those present in mouse or human CSF (2 mM; ref. 49). In the presence of 2-DG, excitatory layer V pyramidal neurons fired significantly fewer APs at a given level of current injection (Figure 1, A and C; see Supplemental Table 1 for all linear mixed model [LMM] statistical results), while GABAergic interneurons were not affected (Figure 1, B and D). Consistent with reduced excitability of glutamatergic excitatory neurons following 2-DG perfusion, rheobase (current injection required to elicit the first AP) was increased in excitatory neurons but was not affected in GABAergic interneurons (Figure 1, E and H). The decrease in the intrinsic excitability of excitatory neurons was associated with a decrease in membrane resistance, which was not observed in GABAergic interneurons (Figure 1, F and I). No change in resting membrane potential was observed in either cell type upon application of 2-DG (Figure 1, G and J). 


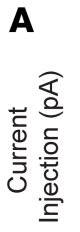

Excitatory Neuron

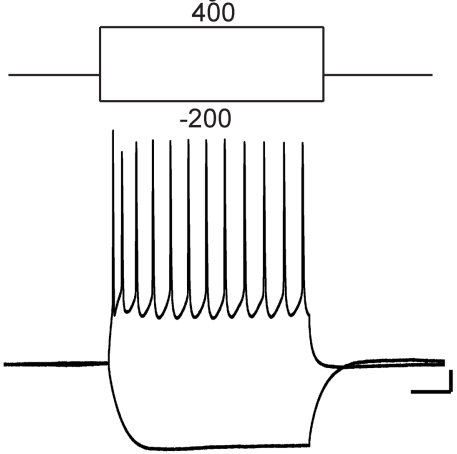

ஸे

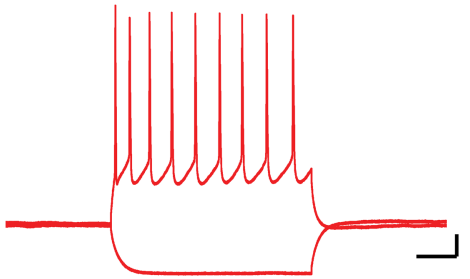

C

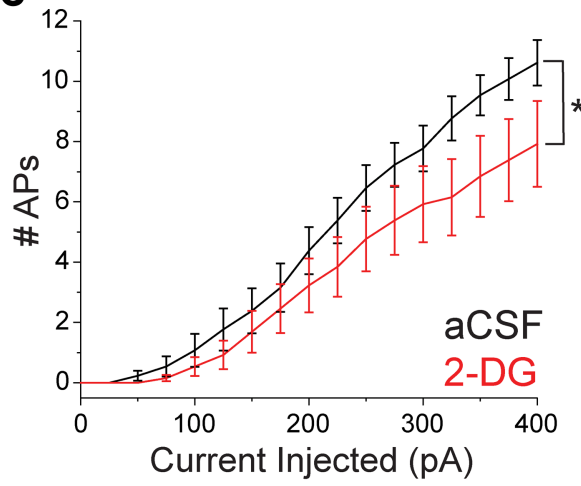

B Inhibitory Interneuron

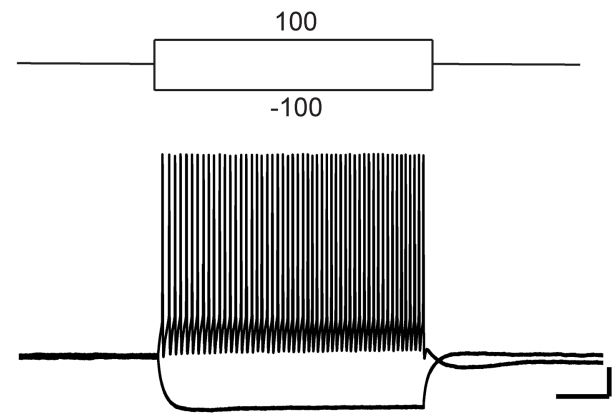

D

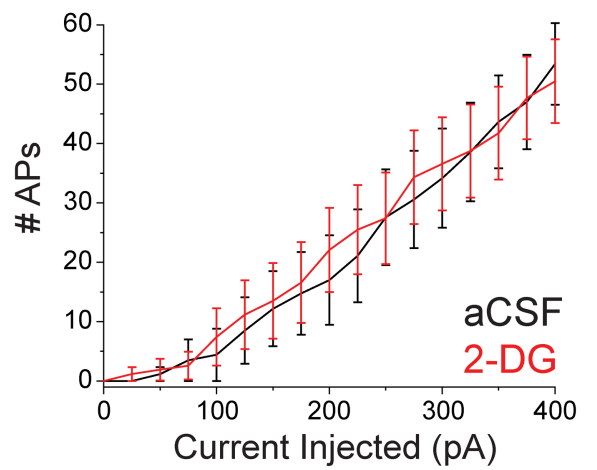

E

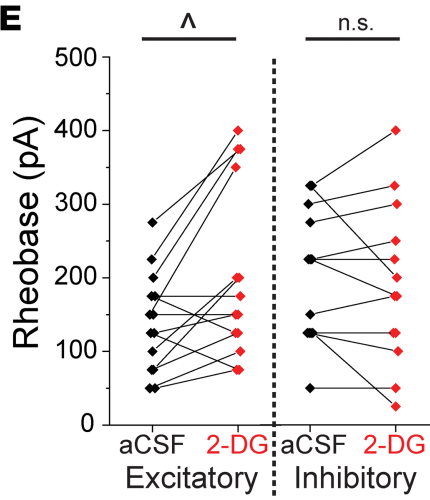

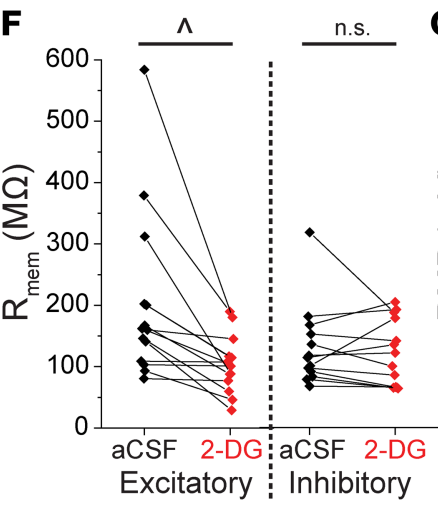
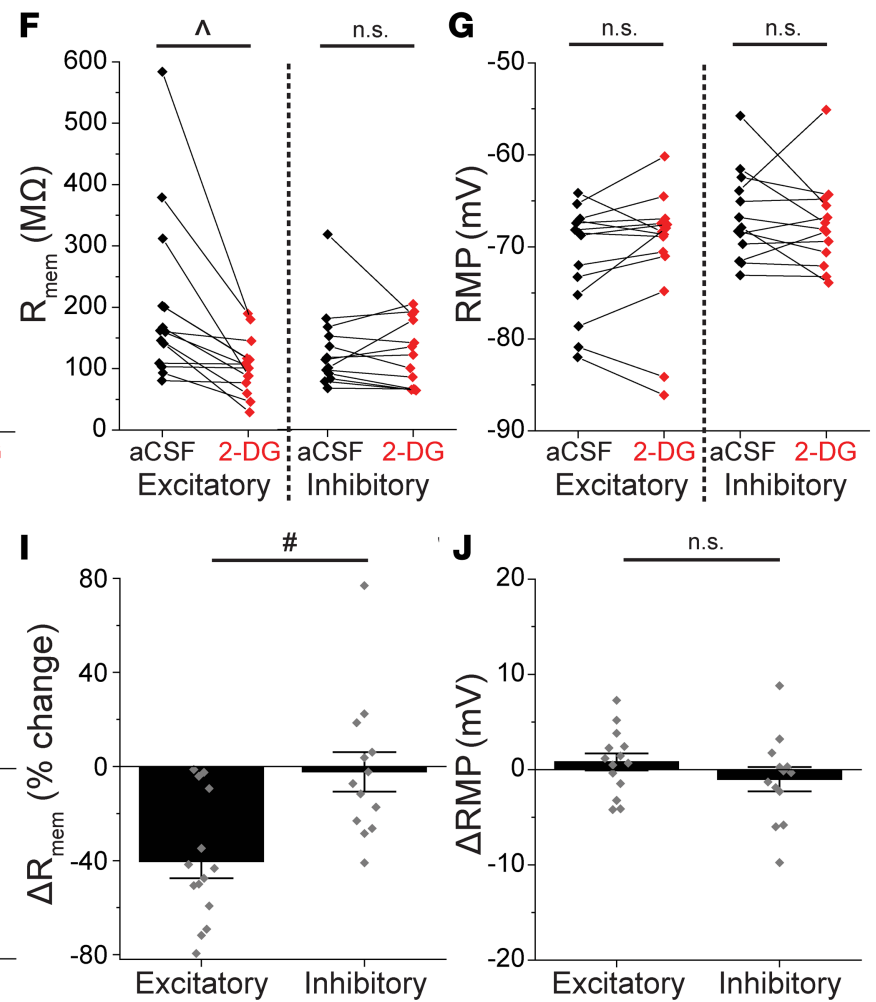

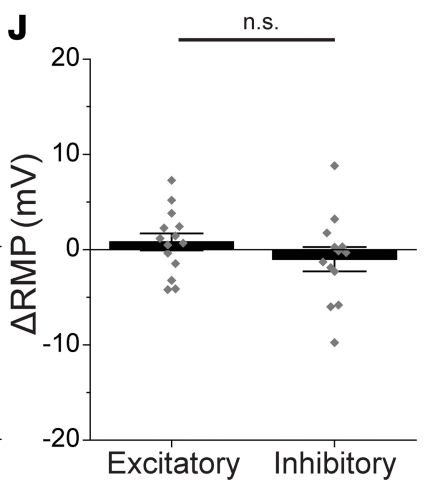

Figure 1. In vitro 2-DG treatment decreases the intrinsic excitability of excitatory pyramidal neurons. (A and B) Representative traces following current injection into layer $\mathrm{V}$ cortical excitatory pyramidal neurons (A) or interneurons (B) before (black) or after (red) treating the cortical slice with 2-DC for 10 minutes. (C and D) Input-output curves from excitatory pyramidal neurons (C) and inhibitory interneurons (D). (E-G) Rheobase (E, current injection required to fire the first action potential), membrane resistance $(\mathbf{F})$, and resting membrane potential (RMP) (G) before (black) and after (red) 2-DG treatment in each cell type. (H) $\Delta$ Rheobase (rheobase in 2-DG versus baseline) in excitatory neurons and interneurons. (I) Percentage change in membrane resistance of excitatory neurons and interneurons following 2-DC treatment. (J) Change in RMP in excitatory neurons and interneurons in 2-DC. Error bar $=$ SEM. $n=14$ excitatory neurons from 9 animals, 13 inhibitory interneurons from 10 animals. LMM: *indicates 1.96 $<t<-1.96$ (effect: interaction between current injection and 2-DG); "indicates $1.96<t<$ -1.96 (effect: cell type). ${ }^{\wedge} P<$ 0.05 by 2 -sample $t$ test. n.s., not significant.

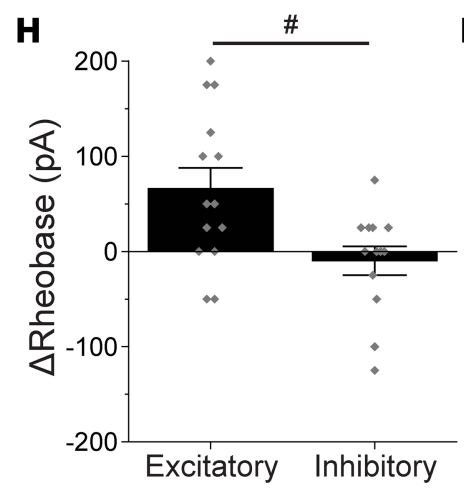


To control for reduced $2 \mathrm{mM}$ glucose in 2-DG-aCSF, these experiments were repeated in low-glucose aCSF (LG-aCSF: $2 \mathrm{mM}$ glucose, $8 \mathrm{mM}$ sucrose [a metabolically inert sugar]). LG-aCSF had no effects on AP firing, input-output curves, or membrane resistance in either excitatory neurons or inhibitory interneurons (Supplemental Figure 2). This demonstrates that simply lowering glucose to $2 \mathrm{mM}$ is not sufficient to alter neuronal excitability. Together, these experiments show that inhibition of glycolysis has cell type-specific effects on neuronal excitability.

2-DG ameliorates CCI-induced increases in synaptic excitation onto GABAergic interneurons. Acutely after TBI, glycolytic activity and neuronal activity are aberrantly increased (35). We hypothesized that TBI results in increased synaptic excitation of GABAergic interneurons in the peri-injury cortex acutely after injury. If so, this hyperexcitation may contribute to later cell death or dysfunction of $\mathrm{PV}^{+}$(and other GABAergic) interneurons (see Discussion). To test this hypothesis, we utilized CCI to model focal, contusional TBI in adult male mice. Briefly, a craniectomy was made over the left sensorimotor cortex and an impact was delivered to generate a moderate-to-severe injury ( $3 \mathrm{~mm}$ impactor probe diameter, $3.5 \mathrm{~m} / \mathrm{s}$ velocity, $400 \mathrm{~ms}$ dwell time, $1 \mathrm{~mm}$ impact depth; ref. 44). Acute cortical slices were prepared from sham- and CCI-injured brains 3 days following injury and whole-cell recordings were made from fast-spiking GABAergic interneurons within $200 \mu \mathrm{m}$ of the lateral edge of the CCI lesion. Interneurons were identified with GFP labeling in layer V-VI cortex in G42 mice, which, as above, were primarily $\mathrm{PV}^{+}$(Supplemental Figure 1). Neurons were voltage clamped at $-70 \mathrm{mV}$ to isolate spontaneous excitatory postsynaptic currents (sEPSCs). Consistent with our hypothesis, there was an increase in the mean frequency of sEPSCs after CCI compared with sham (Figure 2B). CCI also resulted in a significant leftward shift in the sEPSC inter-event interval cumulative distribution (Figure 2C). There was a trend towards an increase in sEPSC amplitude, without statistical significance based on LMM or 2-sample Kolmogorov-Smirnov test (Figure 2, F-G).

Because glucose utilization is elevated acutely after TBI and we had previously observed specific effects of 2-DG on excitatory (but not inhibitory) neuronal activity, we assessed whether 2-DG application to the acute slices would reduce injury-induced excitation of GABAergic interneurons. When 2-DG-aCSF was applied to acute cortical slices prepared 3 days following CCI, the mean frequency of sEPSCs onto interneurons was reduced to approximately sham levels (Figure 2B), corresponding with a rightward shift of the sEPSC inter-event interval cumulative distribution (Figure 2D). Application of 2-DG to sham tissue did not significantly affect sEPSC frequency. There was, however, a large variability in the response to 2-DG treatment in sham animals and a trend toward more frequent excitatory events (data not shown), demonstrating unique effects of 2-DG in sham- versus CCI-injured brain. 2-DG treatment also resulted in a leftward shift of the cumulative event amplitude following CCI (Figure 2H), although the comparisons of the mean amplitude by LMM and cumulative distributions by 2-sample Kolmogorov-Smirnov test did not reach statistical significance. Overall, these results suggest that GABAergic interneurons receive increased excitation in the days following TBI and that this effect is attenuated by acute application of 2-DG.

Acute 2-DG treatment attenuates epileptiform activity in cortical brain slices following CCI. Next, we tested whether 2-DG application attenuates network-level cortical hyperexcitability, which is observable in field excitatory postsynaptic potentials (fEPSPs) as early as 2 weeks following TBI (17), and is robustly present by 3-5 weeks following TBI. Brain slices were prepared from mice 3-5 weeks after CCI or sham surgery and electrical stimulation was delivered to ascending cortical inputs to evoke fEPSPs. In sham animals, fEPSPs were brief and of low amplitude. Slices taken 3-5 weeks after CCI, however, exhibited stimulus-evoked fEPSPs with epileptiform activity (increased amplitude, duration, area, and coastline), consistent with network hyperexcitability (ref. 20; Figure 3A). As shown previously in other slice models of epileptiform activity (40), in vitro application of 2-DG markedly reduced the percentage of stimulus-evoked fEPSP traces that exhibited epileptiform activity after CCI from $90 \%$ to $18 \%$ (Figure 3B). Additionally, 2-DG treatment resulted in field potentials with significantly decreased area (Figure 3C) and high-frequency activity as measured by fEPSP coastline (Figure 3D). In brain slices from sham-injured animals, 2-DG-aCSF did not significantly change the area or coastline of the fEPSPs, or the percentage of epileptiform traces. In low-glucose conditions (LG-aCSF), post-CCI epileptiform activity was not attenuated (Supplemental Figure 3). These results show that in vitro application of the glycolytic inhibitor 2-DG rapidly attenuates cortical network hyperexcitability 3-5 weeks after TBI.

In vivo 2-DG treatment attenuates epileptiform cortical activity following CCI. We next hypothesized that in vivo 2-DG treatment during a critical period following TBI reduces subsequent cortical hyperexcitability. To test this hypothesis, we treated mice with $2-\mathrm{DG}$ throughout the first week after injury $(250 \mathrm{mg} / \mathrm{kg}$ or vehicle intraperitoneally, once daily for 7 days following CCI or sham surgery) and assessed network 
A

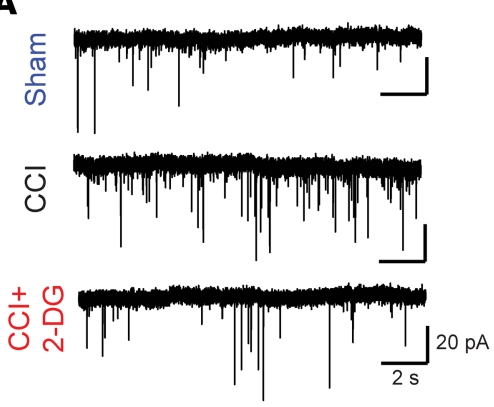

E

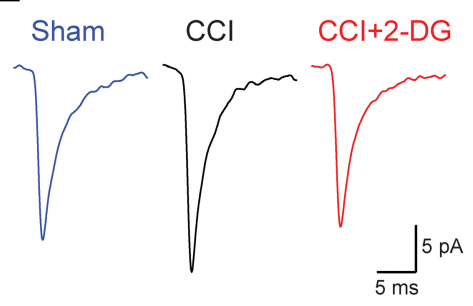

B

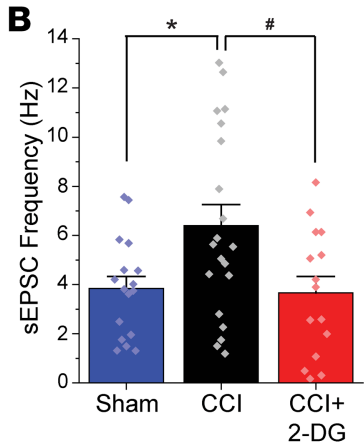

$\mathbf{F}$

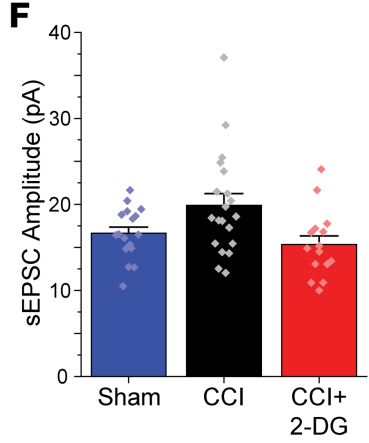

C

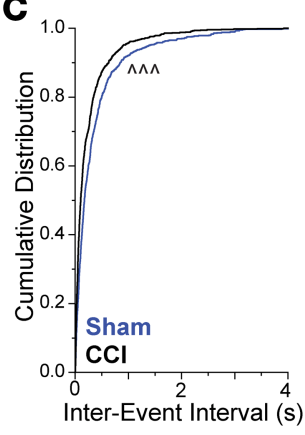

G

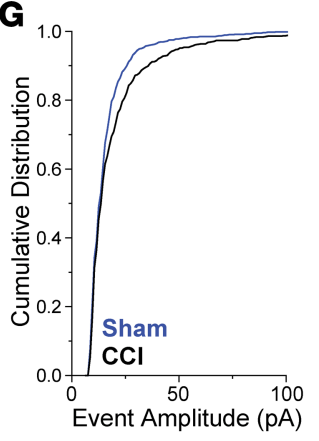

D
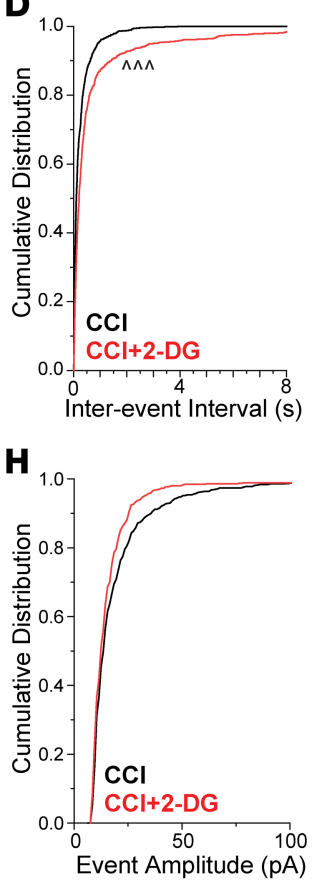

Figure 2. In vitro 2-DC attenuates CCI-induced increases of excitatory synaptic activity onto interneurons 3 days after injury. (A) Representative sEPSC traces from acute cortical slices from sham or CCI-injured animals, and a representative trace from a CCI slice following 10 minutes of 2-DC wash-on. (B) Mean sEPSC frequencies. (C and D) Cumulative distributions of inter-event interval generated from 100 random events per recorded cell. (E) Representative events from each condition, generated by averaging 100 events from a representative trace. (F) Average event amplitude. (G and $\mathbf{H})$ Cumulative distributions of event amplitude generated from 100 randomly selected events per cell. Error bar $=$ SEM. $n=15-20$ cells from 4 animals/group. LMM: ${ }^{*}$ indicates $1.96<t<-1.96$ (effect: CCI); ${ }^{\#}$ indicates $1.96<t<-1.96$ (effect: interaction between CCI and 2-DC). ${ }^{\wedge \wedge} P<1 \times 10^{-5}$ by 2 -sample Kolmogorov-Smirnov test with correction for multiple comparisons.

hyperexcitability utilizing cortical field recordings. Acute cortical brain slices were prepared 3-5 weeks following the initial surgery (2-4 weeks following the end of 2-DG treatment), when epileptiform activity is expected in the CCI animals (20). As predicted, sham-injured slices from both treatment groups had normal fEPSPs (Figure 4, A-B). Consistent with previous studies, acute cortical brain slices from vehicle-treated CCI-injured animals exhibited epileptiform stimulus-evoked fEPSPs (Figure 4C). However, in vivo 2 -DG treatment following CCI dramatically attenuated epileptiform fEPSPs (from $95 \%$ to $13 \%$ ) in CCI-injured animals (Figure 4, D-E). Area and coastline measurements of the evoked fEPSPs following CCI also exhibited significant attenuation with in vivo 2-DG treatment (Figure 4, F-G).

CCI also disrupts the input/output (I/O) relationship of the cortical network. Cortical I/O responses were assessed by comparing fEPSP area evoked by $1 \times$ and $2 \times$ threshold stimulation. In the uninjured brain, cortical networks generated graded output in response to increased stimulation. After CCI, however, cortical networks generated all-or-none responses and lacked graded output in response to stimulation (Figure $4 \mathrm{H}$ ). In vivo 2-DG treatment partially restored the disrupted I/O relationship, resulting in graded fEPSP amplitude and area in response to increased stimulation. 2-DG treatment had a large effect size on the I/O relationship ( 0.54 compared with the effect of CCI, -0.81$)$ but did not reach statistical significance by LMM, likely due to the high degree of variability (Figure $4 \mathrm{H}$ ). Together, these experiments show that in vivo 2-DG treatment during the first week after injury attenuates network hyperexcitability and reduces long-term TBI-associated cortical network dysfunction.

In vivo 2-DG treatment transiently affects systemic glucose metabolism, but does not affect body temperature. Because systemic metabolic changes, such as hypothermia, may have independent neuroprotective effects after TBI, we assessed the effects of in vivo 2-DG on whole-body metabolism. After a single 2-DG injection ( $250 \mathrm{mg} / \mathrm{kg}$, intraperitoneal) in fasted animals, blood glucose levels were transiently increased (Figure 4I) and $\beta$-hydroxybutyrate (a ketone body) levels were transiently decreased (Figure $4 \mathrm{~J}$ ), consistent with decreased glucose utilization and increased ketosis. These changes in blood glucose and $\beta$-hydroxybutyrate returned to baseline by 4 hours after injection. There was also a transient 

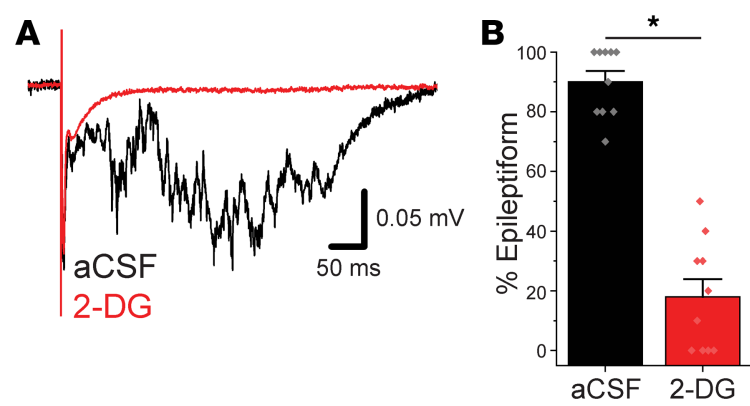
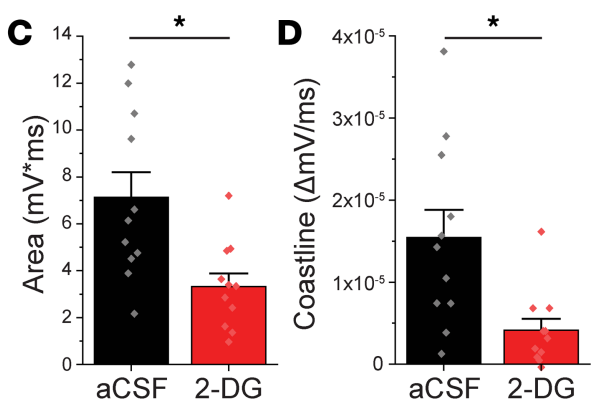

Figure 3. Acute 2-DG treatment decreases epileptiform activity in vitro following CCI. (A) Representative stimulus-evoked field potentials in an acute cortical slice from a CCI-injured animal before (black) and after (red) local perfusion of 2-DG for 30 minutes. (B) Percentage of traces with epileptiform activity after CCI. (C and D) Area (C) and coastline (D) of the field potentials in baseline aCSF and 2-DG-aCSF in CCI-injured slices. Error bar $=$ SEM. $n=10$ slices from 6 animals. LMM: *indicates $1.96<t<-1.96$ (effect: interaction between CCI and 2-DG)

decrease in home cage locomotor activity that resolved to vehicle levels after the first 6 hours after injection (Figure 4K). Importantly, there was no change in flank temperature in 2-DG-injected animals versus vehicle-injected controls (Figure 4L), suggesting that hypothermia does not contribute to the neuroprotective effect of 2-DG.

Then, daily 2-DG injections were performed in naive animals for 1 week to parallel the in vivo dosing regimen after CCI. Animals were assessed at rest, immediately before their daily 2-DG injection ( 24 hours following their most recent injection). There were no changes observed in blood glucose, blood $\beta$-hydroxybutyrate, flank temperature, body weight, or daily food intake between vehicle- and 2-DG-treated animals across the entire week of treatment (Supplemental Figure 4). For all experiments reported in this study, in vivo treatment with 2-DG did not affect animal weight or recovery from surgery. These data suggest that there were no significant adaptive metabolic changes across the week-long in vivo 2-DG treatment.

In vivo 2-DG treatment prevents CCI-induced dysregulation of excitatory and inhibitory synaptic activity. TBI causes significant disruption of cortical synaptic activity including increased glutamatergic excitation and reduced GABAergic inhibition of excitatory cortical neurons (20), accordant with network hyperexcitability. In order to test whether in vivo 2-DG treatment ameliorates post-CCI changes in synaptic activity, we recorded spontaneous excitatory and inhibitory postsynaptic currents onto perilesional layer V pyramidal neurons 3-5 weeks following CCI. Consistent with previous studies, CCI induced a significant increase in mean sEPSC frequency (Figure 5A), resulted in a significant leftward shift in the sEPSC inter-event interval cumulative distribution, and had no effect on sEPSC amplitude (Figure 5C). Inhibitory synaptic transmission was decreased by $\mathrm{CCI}$, again consistent with previous studies. CCI decreased mean spontaneous inhibitory postsynaptic current (sIPSC) frequency (Figure 5G), caused a significant rightward shift in the SIPSC inter-event interval cumulative distribution, and significantly increased mean sIPSC amplitude (Figure 5I).

Consistent with its effects on network hyperexcitability, in vivo 2-DG treatment significantly attenuated CCI-induced increases in excitation. 2-DG treatment reduced mean sEPSC frequency (Figure 5, A and E) and caused a significant rightward shift in the sEPSC inter-event interval cumulative distribution (Figure 5B), with no effect on sEPSC amplitude (Figure 5, C and D). In vivo treatment with 2-DG also restored inhibitory synaptic transmission after CCI. 2-DG had a large, but not significant, effect on mean sIPSC frequency (Figure 5G), caused a significant leftward shift in the sIPSC inter-event interval cumulative distribution (Figure 5H), significantly reduced mean sIPSC amplitude (Figure 5I), and caused a leftward shift in the cumulative distribution of sIPSC amplitude (Figure 5J). Interestingly, we noted a difference in the distribution of mean sIPSC frequency following CCI; in cortical pyramidal cells recorded after sham injury, there was consistently a population of neurons ( 5 out of 25 cells in the sham + vehicle group) that received sIPSCs at a high rate $(>12 \mathrm{~Hz})$. This population was absent after CCI ( 0 out of 21 cells in CCI + vehicle) but was present after in vivo 2-DG treatment (7 out of 21 cells in CCI + 2-DG), suggesting that CCI causes a loss of robust network inhibition that is prevented by 2-DG treatment. Together, these data demonstrate that in vivo 2-DG treatment ameliorates network deficits in spontaneous excitatory and inhibitory neurotransmission caused by CCI. 
A

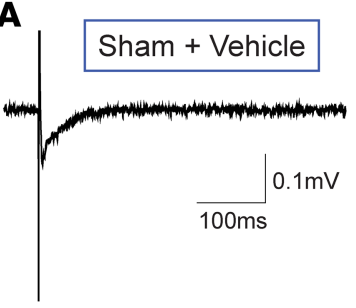

E
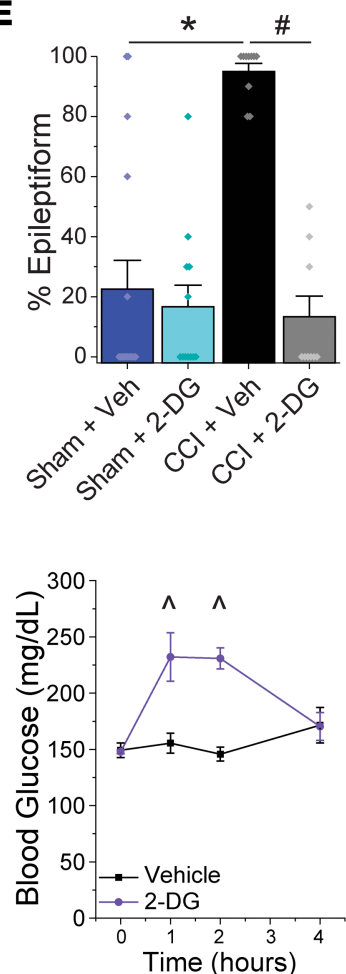

B

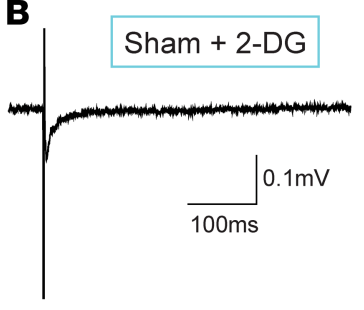

$\mathbf{F}$
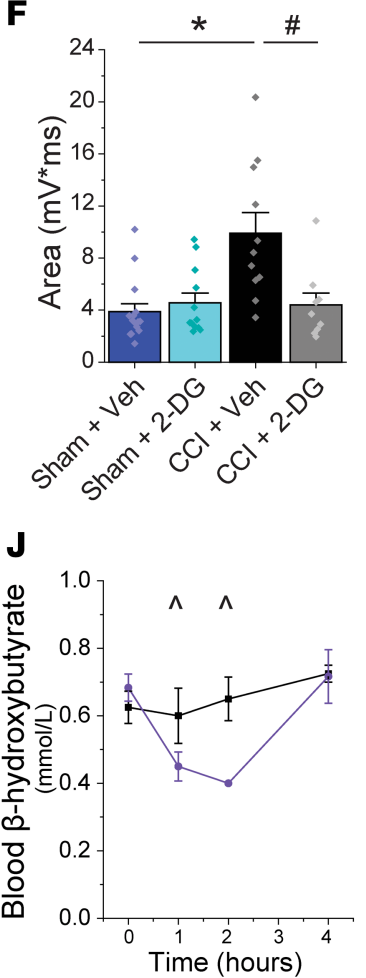
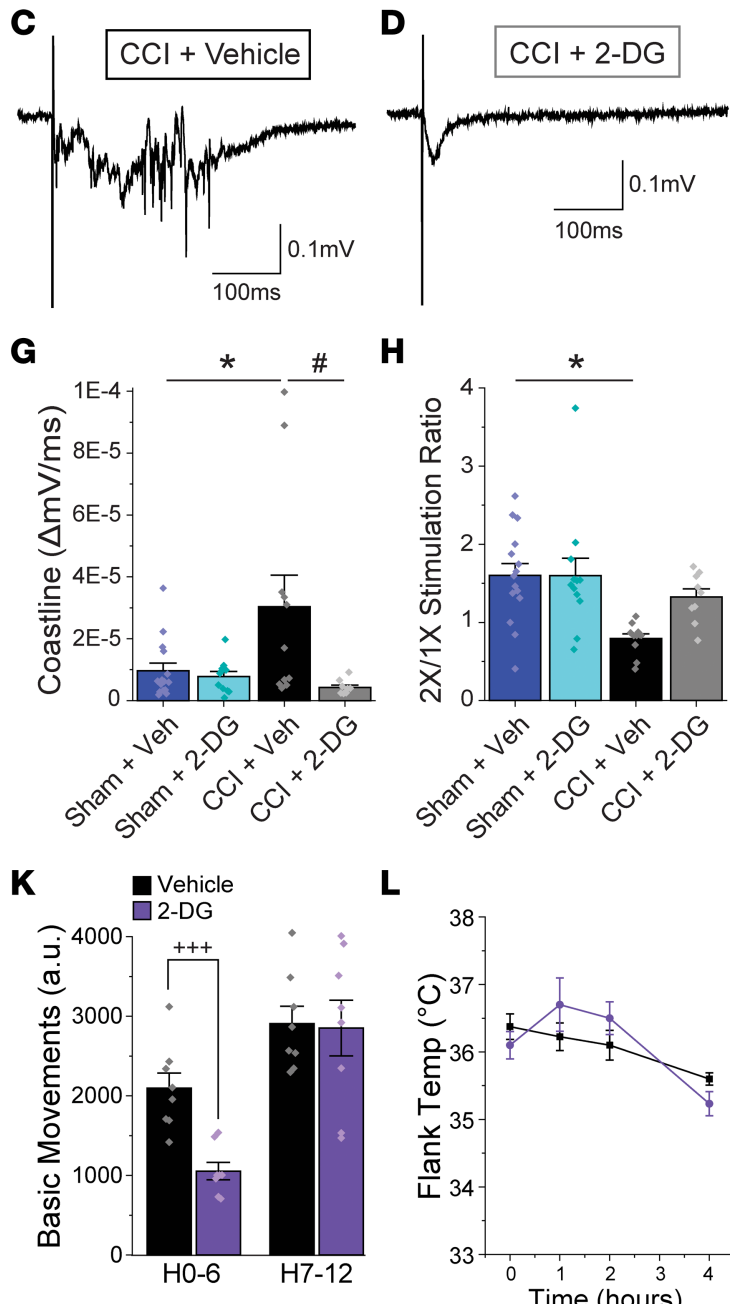

$\mathbf{L}$

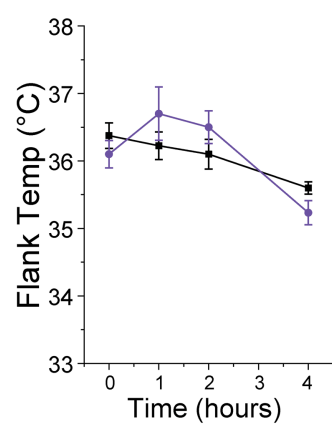

Figure 4. In vivo 2-DG treatment following CCI decreases epileptiform activity in acute cortical slices. Animals were given sham or CCI surgery, followed by 1 week of daily intraperitoneal injections of vehicle or 2-DG $(250 \mathrm{mg} / \mathrm{kg})$. (A-D) Representative stimulus-evoked field potentials taken from acute cortical slices 3 to 5 weeks following sham or CCI surgery, with vehicle or 2-DC treatment. (E) Percentage of sweeps per slice exhibiting epileptiform activity. (F and G) Area (F) and coastline (G) of fEPSP traces. (H) Input/output ratios. (I) Blood glucose in naive fasted mice after a single vehicle (black) or 2-DC (purple) injection. (J) Blood ketone body levels (as measured by $\beta$-hydroxybutyrate) after vehicle or 2-DC injection. (K) Basic locomotor activity in vehicle- or 2-DG-treated animals. (L) Flank temperature after vehicle or 2-DG injection. Error bar $=$ SEM. $n=9-15$ slices from 3 animals/group for $f E P S P s . ~ n=4$ vehicle, 6 2-DG animals for metabolism studies. $n=8$ animals/group for locomotor activity. LMM: *indicates $1.96<t<-1.96$ (effect: CCI); ${ }^{*}$ indicates $1.96<t<-1.96$ (effect: interaction between $\mathrm{CCl}$ and 2-DC); ${ }^{\wedge}$ indicates $1.96<t<-1.96$ (effect: 2-DC at each time point). ${ }^{+++} P<0.001$ by 2 -sample $t$ test.

In vivo 2-DG treatment attenuates the loss of PV-expressing interneurons following CCI. Because 2-DG treatment attenuated the development of epileptiform activity and prevented the decrease in sIPSCs after CCI, we hypothesized that it also prevents the loss of PV-expressing interneurons that had been previously reported (20). An additional question that we wished to address was whether CCI caused a loss of PV cells, or merely a loss of PV protein. To test our hypothesis and address the loss of PV cells versus protein, we used a combination of genetic and immunohistochemical approaches. $P V^{c r e}$ mice (50), in which Cre recombinase is expressed under the control of the Pvalb promoter, were crossed with $A i 9$ reporter mice (51), producing $P V^{\mathrm{Cr}} / \mathrm{A} i 9$ mice that express tdTomato (tdT) in cells containing Cre. This provides a genetic label of PV cells, even if the PV protein itself is lost.

$P V^{C r e} / A i 9$ mice underwent $\mathrm{CCI}$ or sham surgery and were treated in vivo with 2-DG or vehicle as described above. Brains were prepared for immunohistochemical analysis 3-5 weeks after CCI. First, lesion volume was approximated across serial sections in CCI animals treated with vehicle or 2-DG. In vivo 2-DG treatment had no significant effect on cavitary lesion volume (2-sample $t$ test; $n=5$ animals/group, $P=0.557)$. Then, the densities of PV-immunolabeled and tdT-labeled neurons were quantified in five 
A
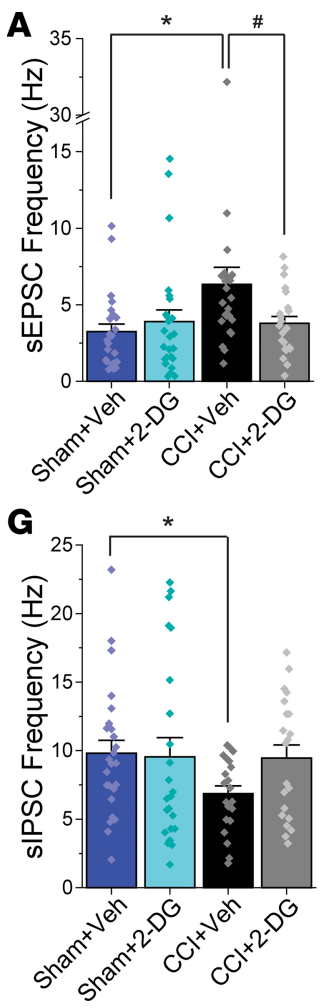

B

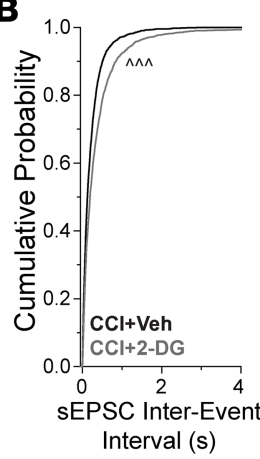

H

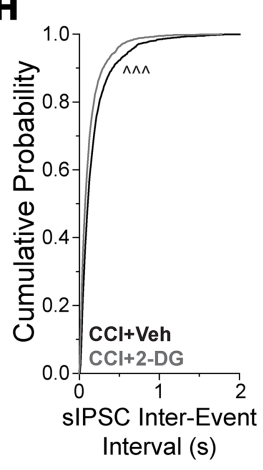

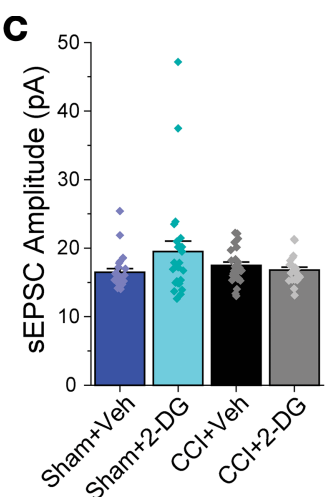
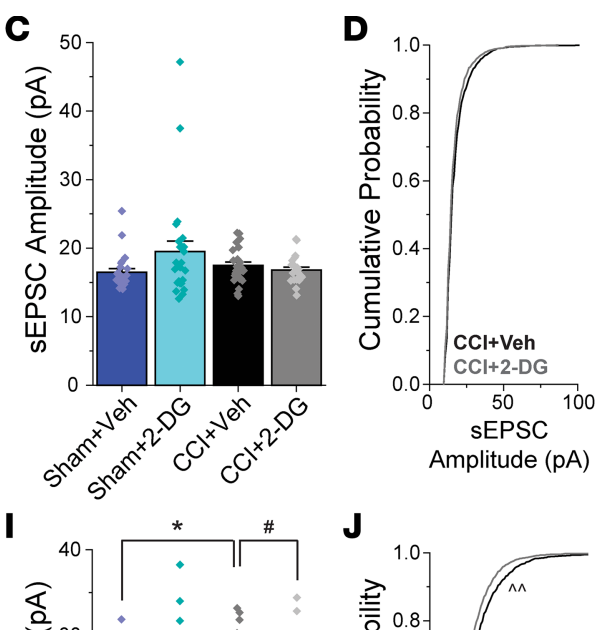$$
\text { J }
$$

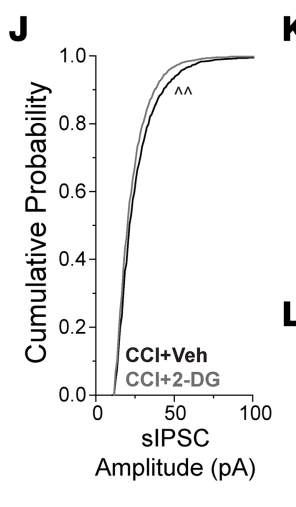

E
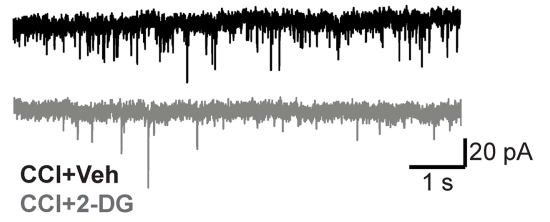

$\mathbf{F}$

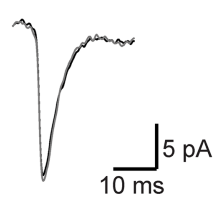

$\mathbf{K}$

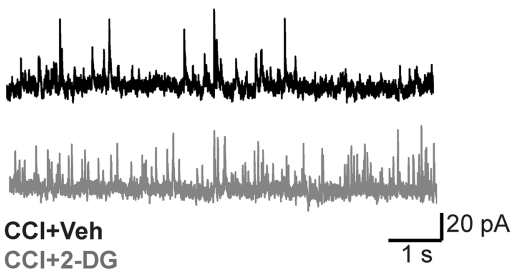

$\mathbf{L}$

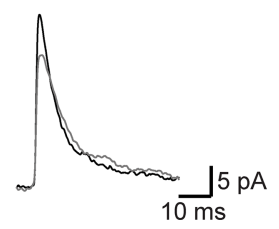

Figure 5. In vivo 2-DC treatment attenuates changes in synaptic activity onto perilesional layer $\mathbf{V}$ pyramidal neurons 3-5 weeks after injury. (A) Mean SEPSC frequency. (B) Cumulative distribution of sEPSC inter-event interval. (C) Mean sEPSC amplitude. (D) sEPSC amplitude cumulative distribution. (E) Representative sEPSC traces from vehicle-treated or 2-DG-treated CCI brains. (F) Representative example of sEPSC events (generated by averaging 100 events from a representative trace). (C) Mean sIPSC frequency. (H) Cumulative distribution of sIPSC inter-event interval. (I) Mean sIPSC amplitude. (J) Cumulative distribution of sIPSC amplitude. (K) Representative sIPSC traces from vehicle-treated or 2-DC-treated CCI brains. (L) Representative example of sIPSC events. Error bar = SEM. $n=21-26$ cells from 5 animals/group. LMM: *indicates $1.96<t<-1.96$ (effect: CCI); ${ }^{*}$ indicates $1.96<t<-1.96$ (effect: interaction between $\mathrm{CCl}$ and $2-\mathrm{DC}$ ). ${ }^{\wedge} \mathrm{P}<\times 10^{-4} ;{ }^{\wedge \wedge} \mathrm{P}<1 \times 10^{-5}$ by 2 -sample Kolmogorov-Smirnov test with correction for multiple comparisons.

100 - $\mu \mathrm{m}$-wide rectangular regions of interest (ROIs) in the cortex lateral to the site of CCI injury (or in isotopic cortex in sham animals). Consistent with previous studies (20), the density of PV-immunolabeled cells was significantly reduced in the cortex of vehicle-treated CCI animals relative to sham (Figure 6, A-E and $\mathrm{G}$ ). $\mathrm{PV}^{+}$cell density was significantly reduced in the first $400 \mu \mathrm{m}$ adjacent to the lesion (Figure 6G). A similar result was seen after counting tdT-labeled neurons (Figure $6 \mathrm{H}$ ), although the decrease was significant only in the first $200 \mu \mathrm{m}$ lateral to the lesion. These results show that CCI causes both a loss of PV cells and a decrease in PV immunoreactivity adjacent to the lesion.

In vivo $2-\mathrm{DG}$ treatment attenuated the loss of $\mathrm{PV}^{+}$neurons relative to vehicle-treated CCI animals (Figure 6, F-G). 2-DG prevented $\mathrm{PV}^{+}$cell loss in the first $300 \mu \mathrm{m}$ adjacent to the lesion. 2-DG did not globally reduce the loss of $\mathrm{tdT}^{+}$neurons, although a significant increase in $\mathrm{tdT}^{+}$neurons was observed in the $100 \mu \mathrm{m}$ closest to the site of injury (Figure 6H), suggesting a focal effect of 2-DG. These results indicate that 2-DG broadly attenuates the loss of PV expression after CCI and focally attenuates the loss of $\mathrm{PV}\left(\mathrm{tdT}^{+}\right)$neurons.

In order to further examine the effects of CCI and 2-DG on $\mathrm{PV}^{+}$cell loss, versus changes in PV protein expression, we assessed the proportion of tdT-labeled neurons that had also been identified as immunopositive for PV. In sham-injured animals, approximately 50\% of tdT-labeled neurons were PV immunopositive. The abundance of tdT-labeled neurons that lacked PV immunolabeling resembles that found in similar studies (52), and is partially attributable to the high threshold that the blinded experimenter used to identify a "PV" cell by immunohistochemistry. The approximately $50 \%$ colocalization of tdT with the PV immunolabel may also result from PV expression below the detection limit of an immunolabeling approach, transient expression of PV during development, or nonspecific Cre expression. After CCI, a significantly higher proportion of tdT-labeled neurons lacked PV immunoreactivity (Figure 6, I-J), with the effect being most 
A Sham + Veh

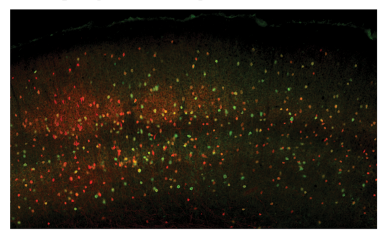

C $\mathrm{CCl}+\mathrm{Veh}$

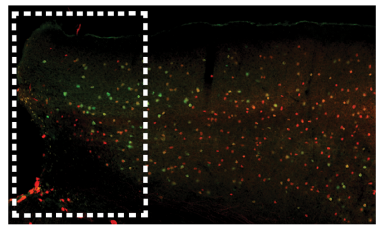

G

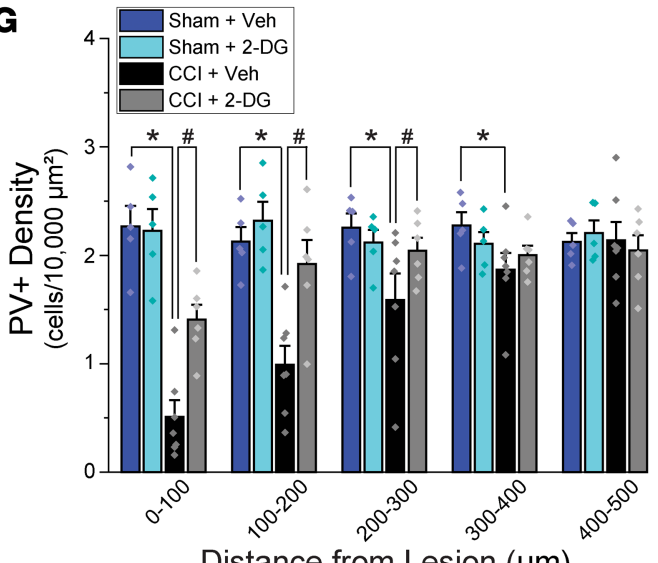

Distance from Lesion ( $\mu \mathrm{m})$
B Sham + 2-DG
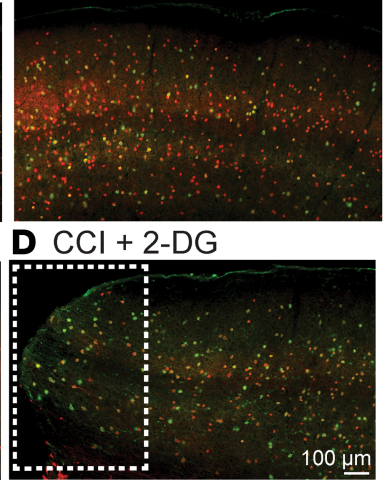
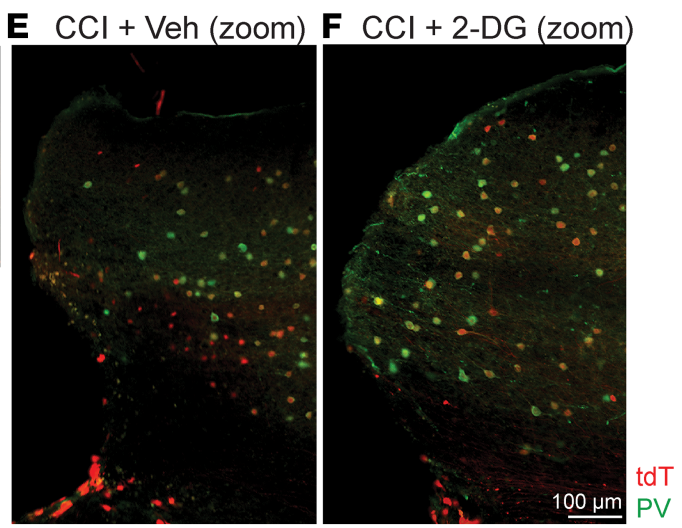

H

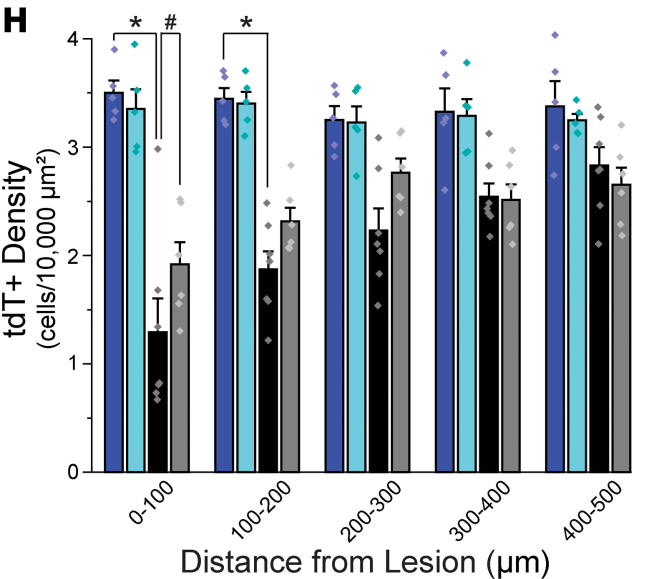

Distance from Lesion $(\mu \mathrm{m})$
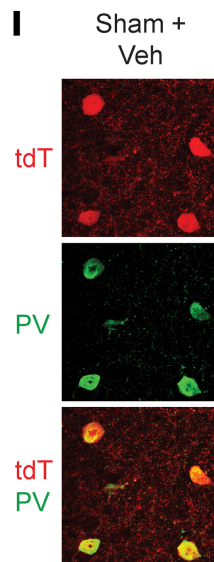

Sham + 2-DG
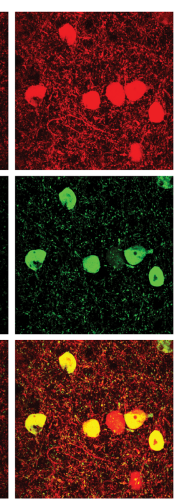
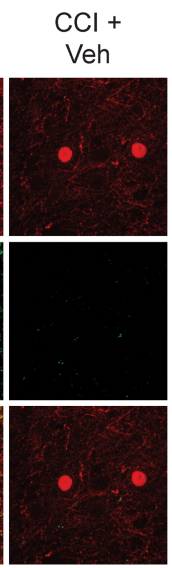
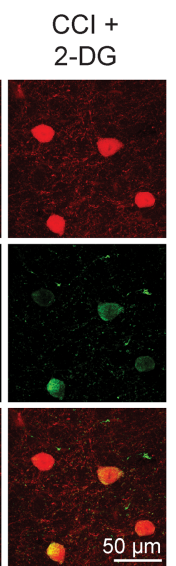

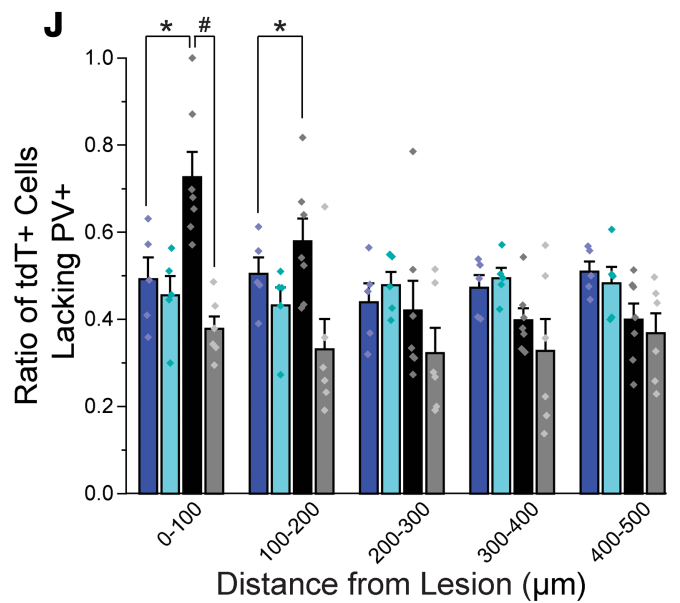

Figure 6. In vivo 2-DG treatment attenuates the decrease in PV+ cells in the perilesional cortex. (A-D) Representative images with PV (green) and tdT (red in PV Cre cells) in animals from the different treatment groups, collected 3-5 weeks after sham or CCI surgery. Scale bars: $100 \mu \mathrm{m}$. (E and F) Zoom-in of perilesional area showing loss of colocalized perilesional $\mathrm{PV}^{+}{ }^{+} \mathrm{td} \mathrm{T}^{+}$cells after $\mathrm{CCI}$. (C) Quantification of $\mathrm{PV}^{+}$cell density in 5 regions lateral to the $\mathrm{CCl}$ cavitary lesion. (H) Quantification of cells identified as $\mathrm{tdT}^{+}$in $P V^{\mathrm{Cr}} / \mathrm{Aig}$ reporter mice. (I) Representative confocal images of cells in the perilesional area with genetic tdT expression (red) and PV immunolabel (green). Scale bar: $50 \mu \mathrm{m}$. (J) The density of tdT+ cells not colocalized with PV immunolabel was divided by the total density of tdT+ cells in each ROI to calculate the ratio reported. Error bar $=$ SEM. $n=5-7$ animals/group ( 3 sections from each animal were analyzed and averaged to generate a single

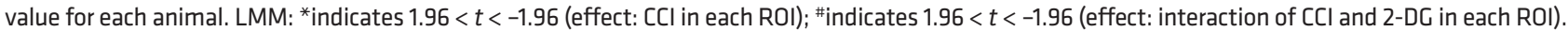

prominent in the first $200 \mu \mathrm{m}$ adjacent to the lesion. This suggests that a subset of $\mathrm{PV}\left(\mathrm{tdT}^{+}\right)$interneurons remain after CCI but lose their expression of PV protein. Because PV is a calcium-binding protein that is necessary for physiologic interneuron AP firing, loss of PV expression is consistent with decreased inhibitory function and cortical hyperexcitability $(53,54)$. In vivo treatment with 2-DG restored the ratio of PV $\left(\mathrm{tdT}^{+}\right)$cells expressing PV to levels seen in sham animals (Figure 6J). Together, these results show that CCI 
causes a loss of $\mathrm{PV}\left(\mathrm{tdT}^{+}\right)$interneurons and reduces $\mathrm{PV}$ expression in the remaining $\mathrm{PV}$ interneurons. In vivo 2-DG treatment broadly increases PV expression in surviving PV interneurons, consistent with enhanced inhibitory function.

\section{Discussion}

2-DG, a hexokinase inhibitor that reduces glycolytic activity, has been previously explored for its potential neuroprotective, anticonvulsant, and anti-epileptogenic properties, but the mechanisms of its rapid actions on neuronal function are unknown. Here, we demonstrate that 2-DG treatment attenuates excitatory, but not inhibitory, cortical neuron excitability. This provides a potential mechanism by which 2-DG mediates its acute anticonvulsant effects. Furthermore, we show that 2-DG has therapeutic potential to treat TBI. While glycolysis is known to be abnormally elevated acutely following brain injury (55), it is unknown whether early metabolic disruptions contribute to chronic cortical hyperexcitability. Using the CCI model of TBI combined with electrophysiological approaches, we find that excitation onto inhibitory interneurons is elevated 3 days after injury and can be attenuated by acute 2-DG treatment. Further, we find that in vivo 2-DG treatment during the first week after TBI prevents epileptiform network activity, restores aspects of synaptic function, and reduces $\mathrm{PV}^{+}$inhibitory interneuron loss associated with CCI. Together, our work provides insight into acute synaptic dysfunction following TBI, implicates glycolysis in the hyperexcitation of inhibitory interneurons after TBI, and shows that in vivo 2-DG treatment reduces posttraumatic synaptic and network dysfunction.

Our findings, summarized above, demonstrate a complex set of synaptic changes following CCI, in both the acute (Figure 2) and chronic (Figure 5) settings. Acutely after TBI (3 days post-CCI), we observe increased excitation of $\mathrm{PV}^{+}$interneurons that we suspect contributes to later inhibitory dysfunction, although our study does not confirm that interneuron loss and dysfunction occur as a direct result of hyperexcitation. Alternatively, increased excitation of interneurons acutely after CCI may represent a compensatory mechanism to increase inhibitory control of cortical activity in the injured brain. If true, however, one would predict that 2-DG's reduction of interneuron hyperexcitation would be detrimental to TBI-related outcomes. We did not observe any increases in adverse events, seizures, or mortality during 2-DG treatment in our studies, consistent with 2-DG's known neuroprotective effects and suggesting that acute hyperexcitation of interneurons is, in fact, pathologic. Further work is required to clarify how early aberrant excitation of interneurons relates to and may result in long-term network pathology, including changes in PV expression, altered interneuron function and synaptic output, and interneuron cell loss.

Next, in the chronic period (3-5 weeks after CCI), we observed changes in both excitatory and inhibitory synaptic activity that were ameliorated with in vivo 2-DG treatment. Consistent with our previous work (20), CCI increased spontaneous synaptic excitation and decreased spontaneous synaptic inhibition onto perilesional layer V pyramidal neurons. Both of these changes were attenuated by in vivo 2-DG treatment, suggesting that 2-DG ameliorates synaptic dysfunction after TBI. In line with the sIPSC frequency data we report, we also found that in vivo 2-DG treatment reduced the loss of PV-expressing interneurons. While changes in inhibitory cell density may contribute to our synaptic findings, changes in inhibitory cell function may also play a role in the overall cortical hyperexcitability evident 3-5 weeks after CCI. For example, loss of PV expression can cause inhibitory dysfunction, hyperexcitability, and worsened seizure phenotype $(53,56,57)$, independent of loss of inhibitory synapses. Additionally, altered chloride transport (reduced KCC2/increased NKCC1 expression; ref. 58-62) and changes in GABA receptor subunit composition and density (63-66) may further contribute to altered inhibitory circuit function. Thus, complex changes in both excitation and inhibition at the synaptic, cellular, and circuit levels result in cortical dysfunction and hyperexcitability after TBI.

Our study demonstrates that in vivo 2-DG treatment significantly ameliorates many of the effects of CCI on synaptic and network activity, and thus strongly supports that modulation of metabolism in the injured brain may have therapeutic potential to improve patient outcomes by maintaining proper network function. Of note, we do not directly demonstrate that in vivo 2-DG treatment reduces brain glycolysis after TBI. 2-DG is well established as an inhibitor of glycolysis (67) across multiple organ systems, animal models, and disease states (68-75). We observe acute changes in blood glucose and ketone body levels (Figure 4, I-J) upon administration of 2-DG, consistent with 2-DG's ability to inhibit glycolysis and decrease glucose utilization. While a recent publication suggests that systemic delivery of 2-DG does not result in appreciable changes in brain hexokinase activity (76), multiple studies demonstrate that 2-DG treatment broadly inhibits glycolysis in whole brain and brain slices $(36,39,40,77)$. Systemic delivery of 2-DG has been shown to 
result in transcriptional changes consistent with reduced glycolysis in the brain, including reductions in hexokinase, PGK-1, and LDH-A expression in the rat hippocampus (39). Furthermore, TBI-associated breach of the blood-brain barrier (78) may allow greater penetration of 2-DG into the brain, resulting in improved inhibition of brain glycolysis. While we observe robust CNS effects after delivering 2-DG post-CCI, it is possible that they are mediated through a peripheral effect of 2-DG or through a mechanism not directly related to hexokinase inhibition.

From a translational standpoint, the therapeutic rationale to reduce glycolysis after TBI is well founded on both preclinical and clinical literature (35). Using PET scanning, studies report that a majority of patients exhibit increased cerebral glucose utilization following severe TBI $(55,79)$ that later develops into a chronic, hypometabolic state (80-82). Additionally, patients with aberrant glucose utilization and network activity during the first week after TBI have worsened outcomes 6 months later (83). Preclinical work from multiple animal models of TBI shows early increases in glucose utilization $(33,84-87)$ that are activity dependent (34). Consistent with focal hyperglycolysis and network dysfunction, our previous studies show heightened glutamate signaling and $\mathrm{PV}^{+}$interneuron loss in the peri-injury cortex (20). Finally, there is abundant evidence supporting the use of the ketogenic diet (which results in decreased glycolysis and increased ketosis) as a strategy to manipulate neural network activity in animals and humans (reviewed in ref. 88-94). While dietary approaches and glycolytic inhibition have shown efficacy in controlling seizure activity, manipulations of glucose metabolism have not yet been harnessed effectively in the context of TBI. In our studies, we did not find evidence of chronic changes in animal health, systemic metabolism, or body temperature (Supplemental Figure 4) over the course of 2-DG treatment. This is important, as long-term exposure to 2-DG ( $>2$ weeks) has been previously associated with cardiotoxicity $(95,96)$ and hypothermia alone may be neuroprotective after TBI (97-99). 2-DG is already in use clinically to limit tumor growth in cancer patients (based on the Warburg effect, which describes increased glycolytic activity in neoplastic cells), and short-term treatments are well tolerated in humans (75). This underscores that short-term use of 2-DG as a disease-modifying agent is a feasible clinical strategy.

A particularly important finding of our study is the cell type-specific coupling of metabolism to neuronal excitability. Our results are concordant with published studies showing that GABAergic interneurons have unique energetic requirements and are known to be enriched with mitochondria for energy production via oxidative phosphorylation (reviewed in ref. 100). Our findings suggest that glutamatergic pyramidal neurons rely on glycolysis to maintain their intrinsic excitability. While glycolysis is traditionally thought to occur mainly in astrocytes (astrocyte-neuron lactate shuttle model; ref. 101), there is substantial evidence that glycolysis also occurs in neurons. Multiple glycolytic enzymes are enriched in neurons relative to astrocytes $(102,103)$ and recent work implicates increased neuronal glycolysis in conditions of heightened energy need $(104,105)$. Thus, increased energetic requirements, glycolytic flux, and cell type-specific neuronal activity may all be interdependent during the acute period following TBI, and deserve further investigation. In future studies, we hope to explore the molecular mechanisms of decreased excitability in excitatory neurons in the presence of 2-DG. We found that decreased excitability in excitatory neurons was associated with significantly decreased membrane resistance, suggesting that the opening of specific ion channels may underly the change in excitability. A possible candidate for this mechanism is an ATP-dependent potassium channel, as $\mathrm{K}_{\mathrm{ATP}}$ channels have been previously shown to link neuronal energy status with excitability (106-108) and have been implicated in network hyperexcitability and epilepsy $(43,109)$. A deeper understanding of metabolic demands following TBI and how different CNS cells utilize energy will aid in the development of metabolic approaches to manipulate neural network activity with greater precision.

In summary, we report that in vitro and in vivo 2-DG treatments attenuate multiple pathologic cellular and network changes following CCI in mice. Future work is needed to investigate the use of 2-DG to prevent injury pathology in other settings, including in diffuse or concussive mechanisms of TBI, in larger animal models, and in pilot clinical trials. Additionally, studies are needed to explore whether reducing cortical hyperexcitability is sufficient to ameliorate patient-oriented outcomes including mortality, seizure frequency/severity in posttraumatic epilepsy, and cognitive/behavioral outcomes. Finally, improving our understanding of the specific energy demands of the neuronal activity, hemodynamic response, and inflammation that occur after TBI will enable us to more effectively harness metabolic manipulations to reduce brain injury-associated pathologies. 


\section{Methods}

\section{Study design}

Basic design. All experiments were hypothesis driven, rigorously designed, and performed in a controlled laboratory environment. Sample size was set based on power analysis calculations using GPower to provide power greater than 0.80 for $\alpha=0.05$ for most experiments. We also utilized standards in the field, i.e., 3 or more animals per group, to ensure proper statistical power.

Randomization and blinding. Mice were randomized to groups within each experiment (sham vs. CCI; vehicle vs. 2-DG). All studies were blinded at the level of data analysis. Data were numerically encoded and analyzed by a trained experimenter blinded to condition. To further prevent bias during analysis, automated analysis approaches were utilized for all synaptic data sets (Figures 2 and 5). After initial analysis was finalized, the blinding was lifted to compare data across groups. Because CCI lesions are easily discernable from sham and in vitro drug applications cannot be reasonably blinded, blinding during data collection of electrophysiological experiments was not feasible (Figures 1-3). Due to constraints of animal tracking, blinding was not performed during in vivo drug injections (Figures 4-6). Immunohistochemical and anatomical experiments were blinded throughout the data collection process (Figure 6).

Exclusion criteria and statistical approach. Each experiment had one primary endpoint based on the experimental design. We did not define rules for stopping data collection outside of humane endpoints based on animal protocols. Data were excluded based on quality control of whole-cell recordings (recordings were excluded when access resistance changed $>20 \%$ during an experiment). Outliers were removed in the context of fEPSP recordings due to inherent variability in the responses evoked with this technique (Figures 3 and 4). Outliers were defined as slices with response area more than 2 standard deviations from the group mean and were excluded from the subsequent analysis. The number of experimental replicates is included in the figure captions and in Supplemental Table 1. The numbers of individual animals, cells, or slices were factored into our analysis using an LMM approach. This approach includes terms to account for inter- and intra-animal variability.

\section{Animals}

All animal procedures were performed in accordance with the Tufts University School of Medicine's Institutional Animal Care and Use Committee. All experiments were performed on adult mice. C57BL/6 mice were obtained from Jackson Laboratories (strain 000664), Charles River Laboratories (strain 027), or bred in-house. $P V^{\text {rre }}$ (stock 017320), Ai9 (stock 07909), and G42 (stock 007677) mice were obtained from Jackson Laboratories. Animals were kept on a standard 12-hour light/12-hour dark cycle and fed ad libitum with regular chow diet and water. Male mice were used for all experiments, unless noted.

\section{CC}

TBI was modeled with CCI, as previously described $(20,110)$. Briefly, 10- to 14-week-old male mice were anesthetized using inhaled isoflurane in oxygen ( $4 \%$ for induction, $2 \%$ for maintenance). Following placement in a Kopf stereotaxic frame, the surgical area was sterilized and a vertical, midline skin incision was made. A 5-mm craniectomy was performed lateral to midline, between bregma and lambda (over the left somatosensory cortex), and the skull flap was removed. The surgical field was flushed with sterile saline throughout the procedure to cool the surgical area during the craniectomy drilling. Impact was performed with a Leica Benchmark Stereotaxic Impactor, using a 3-mm-diameter piston, $3.5 \mathrm{~m} / \mathrm{s}$ velocity, 400-ms dwell time, and 1-mm depth. After the CCI procedure, sutures were used to close the incision. The bone flap was not replaced in order to accommodate swelling after the procedure and to prevent pressure-induced damage to the injury site. Sham animals received the anesthesia and craniotomy drilling, but did not receive the CCI. Animals were singly housed following surgery until time of sacrifice.

\section{In vivo $2-D G$ treatment}

Animals were injected intraperitoneally with $250 \mathrm{mg} / \mathrm{kg}$ 2-DG dissolved in sterile saline to a final injection volume of $200 \mu \mathrm{l}$ per dose. Vehicle injections consisted of $200 \mu \mathrm{l}$ of sterile saline per dose. For the in vivo 2-DG treatment paradigm, the first dose was administered approximately 20 minutes after CCI or sham surgery, and the doses were continued daily for 7 days at the same time of day. Animal weight was recorded daily to monitor animal health. 


\section{Acute brain slice preparation}

Brain slices were prepared as previously described $(20,111)$. Briefly, mice were anesthetized in an isoflurane chamber and then decapitated by guillotine. Brains were rapidly removed and placed in chilled slicing solution (in mM: 234 sucrose, 11 glucose, $24 \mathrm{NaHCO}_{3}, 2.5 \mathrm{KCl}, 1.25 \mathrm{NaH}_{2} \mathrm{PO}_{4}, 10 \mathrm{MgSO}_{4}, 0.5 \mathrm{CaCl}_{2}$ ) equilibrated with $95 \% \mathrm{O}_{2} / 5 \% \mathrm{CO}_{2}$. The brain was then glued to the slicing stage of a Leica VT1200S vibratome and the slicing chamber was filled with chilled slicing solution, again equilibrated with $95 \%$ $\mathrm{O}_{2} / 5 \% \mathrm{CO}_{2}$. Coronal slices ( 300 or $400 \mu \mathrm{m}$ thick) were taken at $0.05 \mathrm{~mm} / \mathrm{s}$ and hemisected. The slices were placed in a chamber filled with aCSF (in mM: $126 \mathrm{NaCl}, 26 \mathrm{NaHCO}_{3}, 3 \mathrm{KCl}, 1.25 \mathrm{NaH}_{2} \mathrm{PO}_{4}, 2 \mathrm{MgCl}_{2}, 2$ $\mathrm{CaCl}_{2}, 10$ glucose) equilibrated with $95 \% \mathrm{O}_{2} / 5 \% \mathrm{CO}_{2}$. The chamber with the acute cortical slices was incubated in a water bath at $34^{\circ} \mathrm{C}$ for 1 hour, and then stored at room temperature until recording.

\section{In vitro treatment of acute cortical slices with 2-DG}

For 2-DG wash-on experiments, 2-DG-aCSF solution was prepared by adding $8 \mathrm{mM}$ 2-DG and removing $8 \mathrm{mM}$ glucose from the standard aCSF recipe (to maintain solution osmolarity, for a final concentration of $2 \mathrm{mM}$ glucose, $8 \mathrm{mM}$ 2-DG). Baseline field or whole-cell recordings were obtained, and then the regular aCSF perfusate was replaced with 2-DG-aCSF for the remaining recordings. For low-glucose wash-on experiments, aCSF containing $2 \mathrm{mM}$ glucose and $8 \mathrm{mM}$ sucrose (an inert sugar) was prepared (LG-aCSF), and LG-aCSF was washed on following baseline field or whole-cell recording collection.

\section{Whole-cell recordings}

Acute cortical slices were placed in a submersion recording chamber of an Olympus BX51 microscope with continuous perfusion of oxygenated $\mathrm{aCSF}$ at $32^{\circ} \mathrm{C}$. Layer $\mathrm{V}$ pyramidal neurons were visually identified with infrared differential interference contrast microscopy, and fast-spiking $\mathrm{PV}^{+}$cortical interneurons were identified based on GFP expression in G42 mice (Jackson Laboratories 007677). Whole-cell recording mode was achieved with a borosilicate glass electrode (3-5 M 2 ) filled with internal solution optimized for each experiment (described below). Access resistance, membrane resistance, and capacitance were monitored throughout each experiment. Cells with greater than $20 \%$ change in access resistance during the experiment were excluded from analysis. Data were collected using an Axon Multiclamp 700B amplifier, Digidata 1440A digitizer, and pClamp software.

For recordings of synaptic activity, the internal solution utilized was, in mM: $140 \mathrm{CsMs}, 10 \mathrm{HEPES}$, $5 \mathrm{NaCl}, 0.2$ EGTA, 5 QX314, 1.8 MgATP, 0.3 NaGTP, $\mathrm{pH}=7.25, \mathrm{mOsm}=290$. The recording electrode was placed within $200 \mu \mathrm{m}$ of the lateral edge of the cavitary cortical lesion. Spontaneous EPSCs and IPSCs were recorded by voltage clamping the cell at -70 and $0 \mathrm{mV}$, respectively, for 2 minutes. Synaptic activity analysis was performed using MiniAnalysis (SynaptoSoft) with the experimenter blinded to experimental group.

For current-injection experiments, the internal solution utilized was, in mM: $120 \mathrm{~K}$ gluconate, $11 \mathrm{KCl}$, 10 HEPES, 10 EGTA, $2 \mathrm{MgCl}_{2}, 2 \mathrm{MgATP}, 0.3 \mathrm{NaGTP}, \mathrm{pH}=7.3, \mathrm{mOsm}=290$. The perfused aCSF also included DNQX $(20 \mu \mathrm{M}), \mathrm{CPP}(10 \mu \mathrm{M})$, and gabazine $(10 \mu \mathrm{M})$ to block synaptic activity. Current-injection steps were applied to each cell (ranging from -200 to 400 pA in 25-pA steps) and cell type was confirmed using AP shape and firing rate.

\section{Field recordings}

Brain slices were placed in an interface chamber perfused with $34^{\circ} \mathrm{C}$, oxygenated aCSF at a rate of 2 $\mathrm{ml} / \mathrm{min}$. A tungsten stimulating electrode was placed at the layer VI-white matter boundary to stimulate ascending white matter tracts. A borosilicate glass micropipette (pulled to a resistance of $\sim 1 \mathrm{M} \Omega$ ) was filled with aCSF and placed in the corresponding area of layer V cortex directly above the stimulating electrode. Recordings were performed in the perilesional cortex (within $100 \mu \mathrm{m}$ of the injury site) or in isotopic cortex in sham animals. Electrical stimulus ( $8-25 \mu \mathrm{A}, 100-\mu$ s pulse length) was delivered using a stimulus isolator (World Precision Instruments) at 30-second intervals. The signal was amplified with a Multiclamp 700A amplifier, digitized with a Digidata 1322A digitizer (sampling rate $=20 \mathrm{kHz}$ ), and recorded with pClamp software (Molecular Devices). Threshold stimulus intensity was defined as the minimal required stimulus to obtain a detectable field response $(\geq 0.05 \mathrm{mV})$. The " $2 \times$ threshold" was defined by doubling the threshold stimulus intensity for a given slice. 


\section{Analysis of field recordings}

Evoked field potentials were analyzed using pClamp software and MATLAB. First, traces were adjusted by subtracting the baseline (the average amplitude directly before the stimulus), imposing a low-pass filter (Bessel 8-pole, 1000-Hz cutoff), and filtering for electrical $60-\mathrm{Hz}$ noise. The area of the field potential was measured by integrating the charge transfer in the first $250 \mathrm{~ms}$ following the stimulus. Coastline measurements describe the relative amount of high-frequency activity in the field response and were measured by summing the distance between each point taken every $50 \mu$ s over a 250 -ms time window following the stimulus. The percentage of epileptiform sweeps for a given slice was determined by counting the number of field potentials that exhibited epileptiform activity (prolonged, high frequency, and high amplitude) out of 10 total sweeps (over a 5-minute period).

\section{Immunohistochemistry}

Animals were transcardially perfused with $100 \mathrm{ml}$ of phosphate-buffered saline (PBS) followed by $100 \mathrm{ml}$ of $4 \%$ paraformaldehyde (PFA) in $0.4 \mathrm{M}$ phosphate buffer (PB). Brains were dissected out, placed in $4 \%$ PFA in PB overnight, and then moved to a $30 \%$ sucrose solution for 3 days. Coronal slices ( $40 \mu \mathrm{m})$ were made on a Thermo Fisher Scientific Microm HM 525 cryostat. For CCI or sham animals, the brains were serially sectioned, so that each collection well contained a representative set of coronal sections throughout the entire CCI lesion, with each section $400 \mu \mathrm{m}$ apart from the next. Slices were washed with PBS and then incubated in blocking buffer (10\% normal goat serum [NGS], 5\% bovine serum albumin [BSA] in PBS with $0.2 \%$ Triton X-100 [PBS-T]) at room temperature for 1 hour. The slices were incubated overnight at $4^{\circ} \mathrm{C}$ with primary antibody in PBS-T with $5 \%$ NGS/1\% BSA. The primary antibodies used were as follows: PV (mouse; Swant PV235; 1:1000 for Figure 6, 1:2000 for Supplemental Figure 1), GFP (chicken; Abcam Ab13970; 1:1000), glutamate decarboxylase 67 (GAD67; mouse; Millipore MAB5406; 1:10,000), somatostatin (SST; rat; Millipore MAB354; 1:100), and calretinin (CR; mouse; Swant 6B3; 1:5000). Slices were then washed with PBS and incubated with secondary antibody (anti-mouse, rat, or chicken antibodies tagged with Alexa 488, Cy3, or 647, applied at 1:500) in PBS-T with 5\% NGS/1\% BSA for 2 hours at room temperature. Finally, slices were rinsed with PBS and mounted, in anatomical order, onto slides using Vectashield with DAPI.

Imaging for cell counting was performed using a Keyence epifluorescence microscope with a $\times 10$ or $\times 20$ optical zoom. Additional images for Figure 6 were taken on a Leica SPE confocal microscope with a $\times 63$ oil objective; additional images for Supplemental Figure 1 were taken on a Nikon A1R confocal microscope with a $\times 40$ oil objective (Olympus). For each experiment, identical laser and capture parameters were utilized across all experimental groups.

\section{Analysis of imaging data}

Image analysis was performed using Fiji/ImageJ software. For Figure 6, five ROIs were drawn lateral to the edge of the cavitary CCI lesion, with each ROI $100 \mu \mathrm{m}$ wide. $\mathrm{PV}^{+}$and $\mathrm{tdT}^{+}$cells were counted independently, by an experimenter blinded to treatment group. The cell counts were then divided by the area of the selected region to find the cell density. The number of $\mathrm{PV}^{+} \mathrm{tdT}^{+}$colocalized cells was determined by comparing the locations of cell bodies selected in the individual $\mathrm{PV}^{+}$and $\mathrm{tdT}^{+}$counting phases. Three sections from each brain (representing the same anatomical regions across all subjects) were quantified and averaged to obtain a single-cell density value per cell type in each ROI in each animal. For Supplemental Figure 1, GFP ${ }^{+}$cells were identified manually in layer V-VI cortex of sections from G42 mice, and then examined for their colabeling with PV, GAD67, SST, or CR immunolabels.

Lesion volume was evaluated in more than 10 serial sections from each animal, mounted and imaged in anatomical order. A blinded experimenter traced the size of the cavitary lesion in each section and the area of missing tissue was recorded. Each area was multiplied by $400 \mu \mathrm{m}$ (the distance between the serial sections) to estimate the lesion volume.

\section{Metabolic studies}

Blood glucose was measured using a OneTouch glucose meter and blood $\beta$-hydroxybutyrate was measured using a PrecisionXtra Ketone Monitor with blood taken from the tail. Flank temperature was measured using a subcutaneous temperature probe (BioMedic Data Systems Implantable Electronic ID Transponders IPTT-300). Food intake was measured by subtracting the final weight from the initial 
weight of the chow during the indicated study period. Home cage locomotor activity was measured utilizing a photobeam-break system (Tufts Behavior Core). Animals were fasted for 16-18 hours before acute measurements following 2-DG injection (Figure 4, I-J and L).

\section{Statistics}

Most experiments used a $2 \times 2$ factorial design. To assay the statistical significance of each factor (fixed effect) and the interaction between factors, we performed LMM analysis (see Supplemental Table 1, supplemental methods, and ref. 112). For comparison of cumulative distributions of inter-event interval or amplitude of synaptic events, we utilized a 2-sample Kolmogorov-Smirnov test with $\alpha$ level corrected for multiple comparisons. For analyzing home cage locomotor activity and lesion volume, we used a 2-sample Student's $t$ test (2-tailed) with $\alpha=0.05$.

\section{Study approval}

All animal procedures were performed in accordance with (and approved by) the Tufts University School of Medicine's Institutional Animal Care and Use Committee. No human subjects or tissues were used in this study.

\section{Drugs and reagents}

Salts were obtained from Sigma-Aldrich or Thermo Fisher Scientific. Glucose was obtained from Thermo Fisher Scientific. 2-DG and DNQX were obtained from Sigma-Aldrich. CPP was obtained from Abcam. Gabazine was obtained from Tocris.

\section{Author contributions}

CGD and JBK conceived of and designed experiments examining 2-DG's effects on neuronal excitability and synaptic activity. CGD, D. Cantu, and JBK conceived of and designed experiments examining 2-DG's effects on epileptiform activity and interneuron density. CGD, JBK, CL, and DK conceived of and designed experiments examining effects of 2-DG on metabolism. JBK performed experiments and analyzed the data for Figures 1, 2, 5, and 6, and Supplemental Figures 2 and 3. D. Cantu performed experiments for Figure 3 and Figure 4, A-H; D. Cantu and JBK analyzed the data for Figure 3 and Figure 4, A-H. JBK and CL performed metabolic experiments and analyzed the data for Figure 4, I-L, and Supplemental Figure 4. D. Croker performed early iterations of the experiments for Figure 6 and developed PV immunolabel quantification strategies. MS performed experiments and analyzed the data for Supplemental Figure 1. FN performed all LMM statistical analysis (Supplemental Tables 1 and 2). MW served as a consultant for experimental design throughout the study and lent expertise in translational implications. JBK and CGD wrote the first draft of the manuscript; comments from all authors were incorporated into the final draft.

\section{Acknowledgments}

We would like to thank Christian Bjorbaek for his helpful feedback on the manuscript and Maribel Rios and Jamie Maguire for their helpful comments. We would also like to thank Dominique Ameroso for her assistance with confocal imaging for Figure 6. This work was supported by the National Institute of Neurological Disorders and Stroke (R21-NS098009, R56-NS076885, R01-NS100076, R21-NS104478 [all to CGD], F31-NS101741 to JBK, and R01-NS107315, R21-NS097922 [both to DK]), the United States Department of Defense (W81XWH-16-ERP-IDA to CGD), the National Institute of Diabetes and Digestive and Kidney Diseases (R01-DK108797 to DK), and the National Institute of Child Health and Human Development (R21-HD098056 to DK).

Address correspondence to: David Cantu or Chris Dulla, 136 Harrison Ave, South Cove 203, Boston, Massachusetts 02111, USA. Phone number: 617-636-3418; Email: davidcantu82@gmail.com (D. Cantu); chris.dulla@tufts.edu (C. Dulla). 
1. Arciniegas DB, Held K, Wagner P. Cognitive impairment following traumatic brain injury. Curr Treat Options Neurol. 2002;4(1):43-57.

2. Frey LC. Epidemiology of posttraumatic epilepsy: a critical review. Epilepsia. 2003;44(s10):11-17.

3. Gupta PK, et al. Subtypes of post-traumatic epilepsy: clinical, electrophysiological, and imaging features. J Neurotrauma. 2014;31(16):1439-1443.

4. Schierhout G, Roberts I. Prophylactic antiepileptic agents after head injury: a systematic review. J Neurol Neurosurg Psychiatry. 1998;64(1):108-112.

5. Jennett WB, Lewin W. Traumatic epilepsy after closed head injuries. J Neurol Neurosurg Psychiatry. 1960;23:295-301.

6. Chiaretti A, De Benedictis R, Polidori G, Piastra M, Iannelli A, Di Rocco C. Early post-traumatic seizures in children with head injury. Childs Nerv Syst. 2000;16(12):862-866.

7. Katayama Y, Becker DP, Tamura T, Hovda DA. Massive increases in extracellular potassium and the indiscriminate release of glutamate following concussive brain injury. J Neurosurg. 1990;73(6):889-900.

8. Yoshino A, Hovda DA, Kawamata T, Katayama Y, Becker DP. Dynamic changes in local cerebral glucose utilization following cerebral conclusion in rats: evidence of a hyper- and subsequent hypometabolic state. Brain Res. 1991;561(1):106-119.

9. Loup V, et al. Combined FISH and PRINS sperm analysis of complex chromosome rearrangement $t(1 ; 19 ; 13)$ : an approach facilitating PGD. Mol Hum Reprod. 2010;16(2):111-116.

10. Golding EM, Robertson CS, Bryan RM. The consequences of traumatic brain injury on cerebral blood flow and autoregulation: a review. Clin Exp Hypertens. 1999;21(4):299-332.

11. Grundl PD, Biagas KV, Kochanek PM, Schiding JK, Barmada MA, Nemoto EM. Early cerebrovascular response to head injury in immature and mature rats. J Neurotrauma. 1994;11(2):135-148.

12. Hiebert JB, Shen Q, Thimmesch AR, Pierce JD. Traumatic brain injury and mitochondrial dysfunction. Am J Med Sci. 2015;350(2):132-138.

13. Sharp DJ, Scott G, Leech R. Network dysfunction after traumatic brain injury. Nat Rev Neurol. 2014;10(3):156-166.

14. Huusko N, Römer C, Ndode-Ekane XE, Lukasiuk K, Pitkänen A. Loss of hippocampal interneurons and epileptogenesis: a comparison of two animal models of acquired epilepsy. Brain Struct Funct. 2015;220(1):153-191.

15. Wei D, et al. Degeneration and regeneration of GABAergic interneurons in the dentate gyrus of adult mice in experimental models of epilepsy. CNS Neurosci Ther. 2015;21(1):52-60.

16. Tóth K, Eross L, Vajda J, Halász P, Freund TF, Maglóczky Z. Loss and reorganization of calretinin-containing interneurons in the epileptic human hippocampus. Brain. 2010;133(9):2763-2777.

17. Hunt RF, Boychuk JA, Smith BN. Neural circuit mechanisms of post-traumatic epilepsy. Front Cell Neurosci. 2013;7:89

18. Staley K. Molecular mechanisms of epilepsy. Nat Neurosci. 2015;18(3):367-372.

19. Hu H, Gan J, Jonas P. Interneurons. Fast-spiking, parvalbumin ${ }^{+}$GABAergic interneurons: from cellular design to microcircuit function. Science. 2014;345(6196):1255263.

20. Cantu D, et al. Traumatic brain injury increases cortical glutamate network activity by compromising GABAergic control. Cereb Cortex. 2015;25(8):2306-2320.

21. Prince DA, Parada I, Scalise K, Graber K, Jin X, Shen F. Epilepsy following cortical injury: cellular and molecular mechanisms as targets for potential prophylaxis. Epilepsia. 2009;50 Suppl 2:30-40.

22. Avramescu S, Nita DA, Timofeev I. Neocortical post-traumatic epileptogenesis is associated with loss of GABAergic neurons. $J$ Neurotrauma. 2009;26(5):799-812.

23. Carron SF, Yan EB, Alwis DS, Rajan R. Differential susceptibility of cortical and subcortical inhibitory neurons and astrocytes in the long term following diffuse traumatic brain injury. J Comp Neurol. 2016;524(17):3530-3560.

24. Buriticá E, Villamil L, Guzmán F, Escobar MI, García-Cairasco N, Pimienta HJ. Changes in calcium-binding protein expression in human cortical contusion tissue. J Neurotrauma. 2009;26(12):2145-2155.

25. Kim JA, et al. Epileptiform activity in traumatic brain injury predicts post-traumatic epilepsy. Ann Neurol. 2018;83(4):858-862

26. Buckmaster PS, Abrams E, Wen X. Seizure frequency correlates with loss of dentate gyrus GABAergic neurons in a mouse model of temporal lobe epilepsy. J Comp Neurol. 2017;525(11):2592-2610.

27. Chen S, Fujita S, Koshikawa N, Kobayashi M. Pilocarpine-induced status epilepticus causes acute interneuron loss and hyper-excitatory propagation in rat insular cortex. Neuroscience. 2010;166(1):341-353

28. Epsztein J, et al. Ongoing epileptiform activity in the post-ischemic hippocampus is associated with a permanent shift of the excitatory-inhibitory synaptic balance in CA3 pyramidal neurons. J Neurosci. 2006;26(26):7082-7092.

29. Kuruba R, Wu X, Reddy DS. Benzodiazepine-refractory status epilepticus, neuroinflammation, and interneuron neurodegeneration after acute organophosphate intoxication. Biochim Biophys Acta Mol Basis Dis. 2018;1864(9 Pt B):2845-2858.

30. Bergsneider M, et al. Metabolic recovery following human traumatic brain injury based on FDG-PET: time course and relationship to neurological disability. J Head Trauma Rehabil. 2001;16(2):135-148.

31. Vespa P, et al. Metabolic crisis without brain ischemia is common after traumatic brain injury: a combined microdialysis and positron emission tomography study. J Cereb Blood Flow Metab. 2005;25(6):763-774.

32. Jalloh I, et al. Glycolysis and the pentose phosphate pathway after human traumatic brain injury: microdialysis studies using 1,2-(13)C2 glucose. J Cereb Blood Flow Metab. 2015;35(1):111-120.

33. Andersen BJ, Marmarou A. Post-traumatic selective stimulation of glycolysis. Brain Res. 1992;585(1-2):184-189.

34. Goel A. Amano K, Hori T, Kawamata T, Okada Y (2016) Repair and prevention of cerebrospinal fluid leakage in transphenoidal surgery: a sphenoid mucosa technique. Neurosurg Rev. 39: 123-131. Neurosurg Rev. 2017;40(1):37.

35. Koenig JB, Dulla CG. Dysregulated glucose metabolism as a therapeutic target to reduce post-traumatic epilepsy. Front Cell Neurosci. 2018;12:350

36. Stafstrom CE, Roopra A, Sutula TP. Seizure suppression via glycolysis inhibition with 2-deoxy-D-glucose (2DG). Epilepsia. 2008;49 Suppl 8:97-100.

37. Bertoni JM. Competitive inhibition of rat brain hexokinase by 2-deoxyglucose, glucosamine, and metrizamide. $J$ Neurochem. 1981;37(6):1523-1528

38. Cisternas P, Martinez M, Ahima RS, William Wong G, Inestrosa NC. Modulation of glucose metabolism in hippocampal neurons 
by adiponectin and resistin. Mol Neurobiol. 2019;56(4):3024-3037.

39. Garriga-Canut M, et al. 2-Deoxy-D-glucose reduces epilepsy progression by NRSF-CtBP-dependent metabolic regulation of chromatin structure. Nat Neurosci. 2006;9(11):1382-1387.

40. Stafstrom CE, Ockuly JC, Murphree L, Valley MT, Roopra A, Sutula TP. Anticonvulsant and antiepileptic actions of 2-deoxyD-glucose in epilepsy models. Ann Neurol. 2009;65(4):435-447.

41. Lee J, Bruce-Keller AJ, Kruman Y, Chan SL, Mattson MP. 2-Deoxy-D-glucose protects hippocampal neurons against excitotoxic and oxidative injury: evidence for the involvement of stress proteins. J Neurosci Res. 1999;57(1):48-61.

42. Lian XY, Khan FA, Stringer JL. Fructose-1,6-bisphosphate has anticonvulsant activity in models of acute seizures in adult rats. J Neurosci. 2007;27(44):12007-12011.

43. Yang $\mathrm{H}$, et al. The antiepileptic effect of the glycolytic inhibitor 2-deoxy-D-glucose is mediated by upregulation of $\mathrm{K}(\mathrm{ATP})$ channel subunits Kir6.1 and Kir6.2. Neurochem Res. 2013;38(4):677-685.

44. Hunt RF, Scheff SW, Smith BN. Posttraumatic epilepsy after controlled cortical impact injury in mice. Exp Neurol. 2009;215(2):243-252.

45. Shao LR, Stafstrom CE. Glycolytic inhibition by 2-deoxy-d-glucose abolishes both neuronal and network bursts in an in vitro seizure model. J Neurophysiol. 2017;118(1):103-113.

46. Zhao YT, Tekkök S, Krnjević K. 2-Deoxy-D-glucose-induced changes in membrane potential, input resistance, and excitatory postsynaptic potentials of CA1 hippocampal neurons. Can J Physiol Pharmacol. 1997;75(5):368-374.

47. Lujan B, Kushmerick C, Banerjee TD, Dagda RK, Renden R. Glycolysis selectively shapes the presynaptic action potential waveform. J Neurophysiol. 2016;116(6):2523-2540.

48. Chattopadhyaya B, et al. Experience and activity-dependent maturation of perisomatic GABAergic innervation in primary visual cortex during a postnatal critical period. J Neurosci. 2004;24(43):9598-9611.

49. Hegen H, Auer M, Deisenhammer F. Serum glucose adjusted cut-off values for normal cerebrospinal fluid/serum glucose ratio: implications for clinical practice. Clin Chem Lab Med. 2014;52(9):1335-1340.

50. Hippenmeyer S, et al. A developmental switch in the response of DRG neurons to ETS transcription factor signaling. PLoS Biol. 2005;3(5):e159.

51. Madisen L, et al. A robust and high-throughput Cre reporting and characterization system for the whole mouse brain. Nat Neurosci. 2010;13(1):133-140.

52. Nichols J, Bjorklund GR, Newbern J, Anderson T. Parvalbumin fast-spiking interneurons are selectively altered by paediatric traumatic brain injury. J Physiol (Lond). 2018;596(7):1277-1293

53. Schwaller B, et al. Parvalbumin deficiency affects network properties resulting in increased susceptibility to epileptic seizures. Mol Cell Neurosci. 2004;25(4):650-663.

54. DeFelipe J, Garcia Sola R, Marco P, del Río MR, Pulido P, Ramón y Cajal S. Selective changes in the microorganization of the human epileptogenic neocortex revealed by parvalbumin immunoreactivity. Cereb Cortex. 1993;3(1):39-48.

55. Bergsneider $M$, et al. Cerebral hyperglycolysis following severe traumatic brain injury in humans: a positron emission tomography study. J Neurosurg. 1997;86(2):241-251.

56. Drexel M, et al. Selective silencing of hippocampal parvalbumin interneurons induces development of recurrent spontaneous limbic seizures in mice. J Neurosci. 2017;37(34):8166-8179.

57. Muñoz-Manchado AB, et al. Diversity of interneurons in the dorsal striatum revealed by single-cell RNA Sequencing and PatchSeq. Cell Rep. 2018;24(8):2179-2190.e7.

58. $\mathrm{Wu} \mathrm{H}$, et al. Melatonin attenuates neuronal apoptosis through up-regulation of $\mathrm{K}(+)-\mathrm{Cl}(-)$ cotransporter $\mathrm{KCC} 2$ expression following traumatic brain injury in rats. J Pineal Res. 2016;61(2):241-250.

59. Bonislawski DP, Schwarzbach EP, Cohen AS. Brain injury impairs dentate gyrus inhibitory efficacy. Neurobiol Dis. 2007;25(1):163-169

60. Hui $\mathrm{H}$, et al. Inhibition of $\mathrm{Na}(+)-\mathrm{K}(+)-2 \mathrm{Cl}(-)$ cotransporter-1 attenuates traumatic brain injury-induced neuronal apoptosis via regulation of Erk signaling. Neurochem Int. 2016;94:23-31.

61. Lu KT, et al. Inhibition of the $\mathrm{Na}^{+}-\mathrm{K}^{+}-2 \mathrm{Cl}^{-}$-cotransporter in choroid plexus attenuates traumatic brain injury-induced brain edema and neuronal damage. Eur J Pharmacol. 2006;548(1-3):99-105.

62. Wang F, et al. NKCC1 up-regulation contributes to early post-traumatic seizures and increased post-traumatic seizure susceptibility. Brain Struct Funct. 2017;222(3):1543-1556.

63. Raible DJ, Frey LC, Cruz Del Angel Y, Russek SJ, Brooks-Kayal AR. GABA(A) receptor regulation after experimental traumatic brain injury. J Neurotrauma. 2012;29(16):2548-2554.

64. Drexel M, Puhakka N, Kirchmair E, Hörtnagl H, Pitkänen A, Sperk G. Expression of GABA receptor subunits in the hippocampus and thalamus after experimental traumatic brain injury. Neuropharmacology. 2015;88:122-133.

65. Raible DJ, et al. JAK/STAT pathway regulation of GABAA receptor expression after differing severities of experimental TBI Exp Neurol. 2015;271:445-456.

66. Huusko N, Pitkänen A. Parvalbumin immunoreactivity and expression of GABAA receptor subunits in the thalamus after experimental TBI. Neuroscience. 2014;267:30-45.

67. Wick AN, Drury DR, Nakada HI, Wolfe JB. Localization of the primary metabolic block produced by 2-deoxyglucose. J Biol Chem. 1957;224(2):963-969.

68. Kalia VK, Prabhakara S, Narayanan V. Modulation of cellular radiation responses by 2-deoxy-D-glucose and other glycolytic inhibitors: implications for cancer therapy. J Cancer Res Ther. 2009;5 Suppl 1:S57-S60.

69. Miwa H, et al. Leukemia cells demonstrate a different metabolic perturbation provoked by 2-deoxyglucose. Oncol Rep. 2013;29(5):2053-2057.

70. Wang A, et al. Glucose metabolism mediates disease tolerance in cerebral malaria. Proc Natl Acad Sci USA. 2018;115(43):11042-11047.

71. Shintani H, Shintani T, Ashida H, Sato M. Calorie restriction mimetics: upstream-type compounds for modulating glucose metabolism. Nutrients. 2018;10(12):E1821.

72. Floberg JM, Schwarz JK. Manipulation of glucose and hydroperoxide metabolism to improve radiation response. Semin Radiat Oncol. 2019;29(1):33-41. 
73. Ingram DK, Roth GS. Glycolytic inhibition as a strategy for developing calorie restriction mimetics. Exp Gerontol. 2011;46(23):148-154.

74. Kane A, et al. Cardioprotective inhibitors of referfusion injury. Probe Reports from the NIH Molecular Libraries Program. Bethesda (MD): National Center for Biotechnology Information (US); 2010-2012 Dec 10 [updated 2013 March 22].

75. Zhang D, Li J, Wang F, Hu J, Wang S, Sun Y. 2-Deoxy-D-glucose targeting of glucose metabolism in cancer cells as a potential therapy. Cancer Lett. 2014;355(2):176-183.

76. Samokhina E, et al. Chronic inhibition of brain glycolysis initiates epileptogenesis. J Neurosci Res. 2017;95(11):2195-2206.

77. Tekkök S, Krnjević K. Long-term potentiation in hippocampal slices induced by temporary suppression of glycolysis. $J$ Neurophysiol. 1995;74(6):2763-2766.

78. Shlosberg D, Benifla M, Kaufer D, Friedman A. Blood-brain barrier breakdown as a therapeutic target in traumatic brain injury. Nat Rev Neurol. 2010;6(7):393-403

79. Bergsneider M, et al. Dissociation of cerebral glucose metabolism and level of consciousness during the period of metabolic depression following human traumatic brain injury. J Neurotrauma. 2000;17(5):389-401.

80. Kawai N, Nakamura T, Tamiya T, Nagao S. Metabolic disturbance without brain ischemia in traumatic brain injury: a positron emission tomography study. Acta Neurochir Suppl. 2008;102:241-245.

81. Shiga T, et al. Loss of neuronal integrity: a cause of hypometabolism in patients with traumatic brain injury without MRI abnormality in the chronic stage. Eur J Nucl Med Mol Imaging. 2006;33(7):817-822.

82. Nakayama N, Okumura A, Shinoda J, Nakashima T, Iwama T. Relationship between regional cerebral metabolism and consciousness disturbance in traumatic diffuse brain injury without large focal lesions: an FDG-PET study with statistical parametric mapping analysis. J Neurol Neurosurg Psychiatry. 2006;77(7):856-862.

83. Vespa PM, et al. Persistently low extracellular glucose correlates with poor outcome 6 months after human traumatic brain injury despite a lack of increased lactate: a microdialysis study. J Cereb Blood Flow Metab. 2003;23(7):865-877.

84. Thomas S, Prins ML, Samii M, Hovda DA. Cerebral metabolic response to traumatic brain injury sustained early in development: a 2-deoxy-D-glucose autoradiographic study. J Neurotrauma. 2000;17(8):649-665.

85. Casey PA, McKenna MC, Fiskum G, Saraswati M, Robertson CL. Early and sustained alterations in cerebral metabolism after traumatic brain injury in immature rats. J Neurotrauma. 2008;25(6):603-614.

86. Zhou J, et al. Temporal changes in cortical and hippocampal expression of genes important for brain glucose metabolism following controlled cortical impact injury in mice. Front Endocrinol (Lausanne). 2017;8:231.

87. Amorini AM, et al. Metabolic, enzymatic and gene involvement in cerebral glucose dysmetabolism after traumatic brain injury. Biochim Biophys Acta. 2016;1862(4):679-687.

88. Rogawski MA, Löscher W, Rho JM. Mechanisms of action of antiseizure drugs and the ketogenic diet. Cold Spring Harb Perspect Med. 2016;6(5):a022780.

89. Yellen G. Ketone bodies, glycolysis, and KATP channels in the mechanism of the ketogenic diet. Epilepsia. 2008;49 Suppl 8:80-82.

90. Milder J, Patel M. Modulation of oxidative stress and mitochondrial function by the ketogenic diet. Epilepsy Res. 2012;100(3):295-303.

91. Boison D. New insights into the mechanisms of the ketogenic diet. Curr Opin Neurol. 2017;30(2):187-192.

92. Rho JM. How does the ketogenic diet induce anti-seizure effects? Neurosci Lett. 2017;637:4-10.

93. Simeone TA, Simeone KA, Stafstrom CE, Rho JM. Do ketone bodies mediate the anti-seizure effects of the ketogenic diet? Neuropharmacology. 2018;133:233-241.

94. Paoli A, Bianco A, Damiani E, Bosco G. Ketogenic diet in neuromuscular and neurodegenerative diseases. Biomed Res Int 2014;2014:474296.

95. Terse PS, et al. 2-Deoxy-D-glucose (2-DG)-induced cardiac toxicity in rat: NT-proBNP and BNP as potential early cardiac safety biomarkers. Int J Toxicol. 2016;35(3):284-293.

96. Minor RK, et al. Chronic ingestion of 2-deoxy-D-glucose induces cardiac vacuolization and increases mortality in rats. Toxicol Appl Pharmacol. 2010;243(3):332-339.

97. Crompton EM, Lubomirova I, Cotlarciuc I, Han TS, Sharma SD, Sharma P. Meta-analysis of therapeutic hypothermia for traumatic brain injury in adult and pediatric patients. Crit Care Med. 2017;45(4):575-583

98. Lewis SR, Evans DJ, Butler AR, Schofield-Robinson OJ, Alderson P. Hypothermia for traumatic brain injury. Cochrane Database Syst Rev. 2017;9:CD001048.

99. $\mathrm{Ma} \mathrm{H}$, et al. Therapeutic hypothermia as a neuroprotective strategy in neonatal hypoxic-ischemic brain injury and traumatic brain injury. Curr Mol Med. 2012;12(10):1282-1296.

100. Kann O. The interneuron energy hypothesis: Implications for brain disease. Neurobiol Dis. 2016;90:75-85.

101. Pellerin L, et al. Evidence supporting the existence of an activity-dependent astrocyte-neuron lactate shuttle. Dev Neurosci. 1998;20(4-5):291-299

102. Lundgaard I, et al. Direct neuronal glucose uptake heralds activity-dependent increases in cerebral metabolism. Nat Commun. 2015;6:6807.

103. Yellen G. Fueling thought: Management of glycolysis and oxidative phosphorylation in neuronal metabolism. J Cell Biol. 2018;217(7):2235-2246.

104. Jang S, et al. Glycolytic enzymes localize to synapses under energy stress to support synaptic function. Neuron. 2016;90(2):278-291.

105. Bas-Orth C, Tan YW, Lau D, Bading H. Synaptic activity drives a genomic program that promotes a neuronal Warburg effect. J Biol Chem. 2017;292(13):5183-5194.

106. Lutas A, Birnbaumer L, Yellen G. Metabolism regulates the spontaneous firing of substantia nigra pars reticulata neurons via KATP and nonselective cation channels. J Neurosci. 2014;34(49):16336-16347.

107. Martínez-François JR, et al. BAD and $\mathrm{K}_{\mathrm{ATP}}$ channels regulate neuron excitability and epileptiform activity. Elife. $2018 ; 7$.

108. Forte N, Medrihan L, Cappetti B, Baldelli P, Benfenati F. 2-Deoxy-D-glucose enhances tonic inhibition through the neurosteroid-mediated activation of extrasynaptic GABA. Epilepsia. 2016;57(12):1987-2000. 
109. Giménez-Cassina A, et al. BAD-dependent regulation of fuel metabolism and K(ATP) channel activity confers resistance to epileptic seizures. Neuron. 2012;74(4):719-730.

110. Mannix RC, et al. Age-dependent effect of apolipoprotein E4 on functional outcome after controlled cortical impact in mice. J Cereb Blood Flow Metab. 2011;31(1):351-361.

111. Hanson E, et al. Astrocytic glutamate uptake is slow and does not limit neuronal NMDA receptor activation in the neonatal neocortex. Glia. 2015;63(10):1784-1796.

112. Aarts E, Verhage M, Veenvliet JV, Dolan CV, van der Sluis S. A solution to dependency: using multilevel analysis to accommodate nested data. Nat Neurosci. 2014;17(4):491-496. 